\title{
COLLISION OF COSMOLOGICAL SHOCK WAVES PRODUCED BY EXPLOSIONS AND FORMATION OF LARGE-SCALE STRUCTURES
}

\author{
SATOSHI YoShioKa AND SATORU IKeUCHI \\ Division of Theoretical Astrophysics, National Astronomical Observatory of Japan \\ Received 1989 June 1; accepted 1990 March 12
}

\begin{abstract}
We investigate the collision process of two cosmological shock waves produced by explosions by twodimensional numerical hydrodynamical calculations in relation to the formation of large-scale structures. The numerical hydrodynamical code is designed to include self-gravity and cooling processes as well as the cosmic expansion.

The structures resulting from the collisions can be classified into four categories by their global appearances: complete merging case, merging case with a dense ring at the intersection of the two shock waves, nonmerging case with a ring, and nonmerging case separated by a dense wall.

From calculations with various parameters, we obtain the parameter dependence of the structures formed. The parameters searched, which affect the resultant structures, are the explosion redshift, the comoving distance between the two explosions, and the explosion energy for the case that two explosions are identical. In addition, we also calculate the cases that the explosion redshifts are different.

From these, we explore the conditions for merging and ring formation, obtain their semianalytical expressions, and discuss the mechanism of ring formation: a ring is not produced by self-gravity, but it results from the cancellation of momenta in the direction perpendicular to the interaction plane at the collision.

In addition, gravitational instability and fragmentation of expanding rings are examined. These results are discussed in relation to the formation of large-scale structures in the universe.

Subject headings: galaxies: formation - hydrodynamics - shock waves
\end{abstract}

\section{INTRODUCTION}

The distribution of galaxies shows us how luminous matter is distributed on large scales. In the last decade, several redshift surveys of galaxies have revealed the three-dimensional distribution of galaxies on scales greater than tens of megaparsecs. In particular, the first result of the extension of the CfA survey showed a bubble-like distribution of galaxies (de Lapparent, Geller, and Huchra 1986): the universe is filled with nearly spherical voids and galaxies seem to be distributed on the surfaces of the voids. Recent progress of the CfA survey has confirmed this impression (Huchra et al. 1988). The first survey in the southern hemisphere has given a similar appearance (da Costa et al. 1988). In addition, the H I redshift survey by Giovanelli and Haynes (1985) has shown the existence of a very long elongated structure in the Pisces-Perseus region.

Most theories for the formation of large-scale structures and galaxies are based upon the idea that these structures in the universe were produced by gravitational growth of primordial Gaussian density perturbations imprinted in the very early universe. They are often called Gaussian models.

On the other hand, non-Gaussian theories have also been proposed. Popular models are the cosmic string theory and the explosion model (Ostriker and Cowie 1981; Ikeuchi 1981). Although the cosmic string theory can explain many observational facts, it is questionable whether the line density needed for galaxy formation will be obtained from the phase transition.

In the explosion model, shock waves produced by explosive energy release from a small number of seeds such as firstgeneration objects (Ostriker and Cowie 1981; Ikeuchi 1981; Yoshioka and Ikeuchi 1988) or superconducting cosmic strings (Ostrịker, Thompson, and Witten 1986) sweep up ambient intergalactic gas into spherical shells, which fragment into new objects. Galaxies form on these large shells, and thus the bubble-like structure of the galaxy distribution is naturally produced. In addition, thermal electrons produced in the blast waves can give rise to the distortion of the cosmic background radiation (Yoshioka and Ikeuchi 1987; Ostriker and Thompson 1987) recently discovered by Matsumoto et al. (1988).

On the basis of the explosion model, Saarinen, Dekel, and Carr (1987) simulated the formation process of structures produced by explosions and the subsequent gravitational clustering by the $N$-body method. They found that the explosion model can reproduce the observed galaxy distribution on scales of a few to a few tens of megaparsecs provided the galaxies formed at a redshift $z \approx 5-10$, although they did not consider the effect of overlapping of gaseous shells.

Several difficulties with the explosion model have been pointed out. The most serious one is the inevitable appearance of anisotropies of the cosmic background radiation (CBR) (Hogan 1984; Vishniac and Ostriker 1986). The temperature of the CBR in the Rayleigh-Jeans part decreases by inverse Compton scattering when the CBR photons pass through hot gas in the blast wave (Zel'dovich and Sunyaev 1969). Fluctuations of the number of explosions in observing beams will result in spatial fluctuations of the CBR temperature. Yoshioka and Ikeuchi (1988) showed that only voids with radius smaller than $7.5 h_{100}^{-1} \mathrm{Mpc}$ can be produced without contradicting the observed isotropy of the CBR, where $h_{100}$ is the Hubble constant in units of $100 \mathrm{~km} \mathrm{~s}^{-1} \mathrm{Mpc}^{-1}$. Yoshioka and Ikeuchi (1987) and Ostriker and Thompson (1987), however, suggested these anisotropies may be erased out by subsequent Thompson scatterings with ionized intergalactic gas if the explosion redshift is larger than $40 \sim 50$. Merging of relatively 
small shells may make the observed large voids. Thus, the overlapping of expanding shells must be explored.

In recent years, several attempts to analyze the overlapping structures of expanding shells have been proposed from the standpoint of view of space division. In Yoshioka and Ikeuchi (1989), we examined the structures which result from the overlapping of shells produced by explosions or negative density perturbations using a three-dimensional Voronoi tessellation. The tessellation was made by a simple Monte Carlo method where mass distribution is represented by particles. The resultant structure was compared with the real structure by utilizing correlation functions of the mass particles and vertices where Voronoi cells intersect. The correlation function of the Voronoi vertices was shown to follow a power law with index $\sim-2$, which is similar to that of clusters (Bahcall and Soneira 1983). Pierre, Shaver, and Iovino (1988) performed calculations similar to ours using a Voronoi tessellation as a minimal model and examined void structure in relation to the distribution of the Lyman- $\alpha$ forest. In addition, van de Weygaert and Icke (1989) have constructed three-dimensional Voronoi tessellation and obtained a similar conclusion regarding the correlation of the Voronoi vertices. Ostriker and Strassler (1989) also calculated structures produced by overlapping of bubbles and compared them with the CfA survey slice. Moreover, Weinberg, Ostriker, and Dekel (1989) also calculated structures produced by overlapping of shells. They assumed clusters form at points where three shells collide and tried various statistical comparisons with the observations. They successfully reproduced the two-point correlation function of clusters as in Yoshioka and Ikeuchi (1989).

However, these simulations do not have a sufficient physical basis; especially, collision processes of cosmological shock waves have not been made clear. Collision processes of two supernova remnants in the interstellar medium have been investigated with two-dimensional hydrodynamical calculations by several authors (Ikeuchi 1978; Jones et al. 1979; Bodenheimer, York, and Tenorio-Tagle 1984; Różyczka, Tenorio-Tagle, and Bodenheimer 1986). In particular, Różyczka et al. succeeded in simulating complicated phenomena with their high-resolution hydrocode. However, selfgravity has not been included in the existing calculations.

In this paper, we investigate the collision process of the cosmological shock waves produced by explosions using numerical hydrodynamical calculations, including self-gravity and cooling as well as cosmic expansion. The aims of this investigation are to resolve the following questions.

1. What structures are formed by the collisions of two cosmological shock waves? What are conditions for merging of the shock waves?

2. How large a structure can be produced by merging of collided shells? How is the structure in the universe produced based on the explosion model?

The plan of this paper is as follows. In $\S$ II, we introduce the method of calculations and the underlying model. In $\S$ III, we show the typical results of calculations and the parameter dependences of structures produced by the collisions. In $\S$ IV, we analyze the numerical results in more detail. In $\S \mathrm{V}$, we examine gravitational instabilities of rings produced by collisions. In $\S \mathrm{VI}$, we constrain the model parameters by comparing the numerical results with observations. In $\S$ VII, we summarize our results and discuss the structure formation in the explosion model.

\section{MODEL AND METHOD OF CALCULATION}

\section{a) Cosmological Model}

We take three cases of model universe: (1) the HD model: a baryon-dominated, flat universe $\left(\Omega=\Omega_{g}=1\right.$, where $\Omega$ is the density parameter of the universe and $\Omega_{g}$ is the fraction of the gas density to the closure density); (2) the LD model: a baryondominated, open universe $\left(\Omega=\Omega_{g}=0.1\right)$, and (3) the DM model: a flat universe dominated by dark matter which is assumed to be distributed homogeneously and treated only as a background gravitational source $\left(\Omega=1\right.$ and $\left.\Omega_{g}=0.1\right)$. Theories of primordial nucleosynthesis in the standard big bang cosmology predict $0.01 \lesssim \Omega_{b} \lesssim 0.1$ (e.g., Yang et al. 1984) and so the cosmological models (2) and (3) may be adequate. However, inhomogeneities created at the quark-hadron phase transition could influence nucleosynthesis and may allow a baryon-dominated, flat universe (model [1]) (Witten 1984; Applegate, Hogan, and Scherrer 1987; Alcock, Fuller, and Mathews 1987). As to model (3), the assumption that dark matter has a role only as a homogeneous background field is valid for relatively early phase of blast waves, though it may not be valid when potential wells of gas shells become deep to affect the motion of dark matter. The fractional density perturbation to the total density by a thin gas shell produced by the explosion is $\sim \Omega_{g}$. Then, this perturbation would go into a nonlinear stage at $z \sim \Omega_{g}\left(1+z_{i}\right)-1$. Thus, the assumption made on the dark matter would break down at early epoch for models of high $z_{i}$ explosions. Throughout the calculations, the Hubble constant is set to $H_{0}=100 \mathrm{kms}^{-1} \mathrm{Mpc}^{-1}$, i.e., $h_{100}=1$.

\section{b) Basic Equations}

We express the equations of gas dynamics in Eulerian coordinate. Let $\rho$ be the gas density, $\boldsymbol{u}$ the gas velocity, $p$ the gas pressure, $\epsilon$ the specific internal energy of the gas, and $\Phi$ the gravitational potential.

The equations of continuity, motion, and energy are, respectively,

$$
\begin{gathered}
\frac{\partial}{\partial t} \rho+\nabla \cdot(\rho \boldsymbol{u})=0, \\
\rho\left[\frac{\partial \boldsymbol{u}}{\partial t}+(\boldsymbol{u} \cdot \nabla) \boldsymbol{u}\right]=-\nabla p-\rho \nabla \Phi, \\
\frac{\partial}{\partial t}(\rho \epsilon)+\nabla \cdot(\rho \epsilon \boldsymbol{u})=-p \nabla \boldsymbol{u}-\Lambda,
\end{gathered}
$$

where $\Lambda$ represents the energy loss rate per unit volume due to radiative cooling and inverse Compton cooling. The gravitational potential is determined by the Poisson equation,

$$
\nabla^{2} \Phi=4 \pi G \rho
$$

where $G$ is the gravitational constant. We take the usual equation of state with the ratio of specific heats $\gamma$,

$$
\epsilon=\frac{1}{\gamma-1} \frac{p}{\rho} .
$$

The above equations are based upon the Newtonian approximation, which is valid since length scales are small compared to the Hubble length $c / H$ in the present situation. In order to treat the cosmological expansion properly, we follow the method of Shandarin (1980) and Shapiro and Struck- 
Marcell (1985). We define a new set of dimensionless, comoving variables, which describe perturbed fluid quantities relative to the mean, adiabatic cosmic expansion as follows:

$$
\begin{array}{rlrl}
\tilde{r}_{i} & =a^{-1}\left(r_{i} / r_{0}\right), & d \tilde{t}=a^{-2}\left(d t / t_{0}\right), & \tilde{\rho}=a^{3}\left(\rho / \rho_{0}\right), \\
\tilde{v}_{i}=a\left(v_{i} / v_{0}\right), & \tilde{\phi}=a^{2}\left(\phi / \phi_{0}\right), & \tilde{p}=a^{5}\left(p / p_{0}\right), \\
\tilde{\epsilon}=a^{2}\left(\epsilon / \epsilon_{0}\right), & &
\end{array}
$$

where $a$ is the scale factor of the universe, $t$ is the time, $r_{i}$ are the spatial coordinates, $\phi$ and $v_{i}$ are the peculiar gravitational potential and velocity, respectively, and all zero-subscripted variables are fiducial values of the corresponding physical quantities. The actual velocity $u_{i}$ can be written as a sum of the average expansion of the universe and this peculiar velocity as follows:

$$
u_{i}\left(t, r_{i}\right)=H(t) \cdot r_{i}+v_{i}\left(t, r_{i}\right),
$$

where $H(t)=\dot{a} / a$ is the Hubble constant. Similarly, the actual gravitational potential $\Phi$ can be written as the sum of the contribution from a homogeneous, isotropically expanding universe and that from the perturbation, as follows:

$$
\Phi\left(t, r_{i}\right)=2 \pi G \bar{\rho} r_{i}^{2} / 3+\phi\left(t, r_{i}\right),
$$

where $\bar{\rho}$ is the mean mass density at time $t$. The scale factor $a$ is related to the dimensionless time $\tilde{t}$ as

$$
a(\tilde{t})=\left(\tilde{t}^{2}-\alpha\right)^{-1}
$$

where

$$
\alpha\left(\boldsymbol{\Omega}_{0}\right)=\left(1-\boldsymbol{\Omega}_{0}\right) / \mathbf{\Omega}_{0} .
$$

The zero-subscripted, dimensional constants are fixed by the present value of Hubble constant $H_{0}$, the present density parameter $\Omega_{0}$, and the parameter $r_{0}$ as follows:

$$
\begin{gathered}
t_{0}=\frac{2}{H_{0} \Omega_{0}^{1 / 2}}, \quad v_{0}=r_{0} / t_{0}, \quad \rho_{0}=3 \Omega_{0} H_{0}^{2} /(8 \pi G), \\
p_{0}=\rho_{0} v_{0}^{2}, \quad \epsilon_{0}=\phi_{0}=v_{0}^{2} .
\end{gathered}
$$

The equations of continuity, motion, and energy are then rewritten to

$$
\begin{gathered}
\frac{\partial}{\partial \tilde{t}} \tilde{\rho}+\tilde{\nabla} \cdot(\tilde{\rho} \tilde{\boldsymbol{v}})=0, \\
\tilde{\rho}\left[\frac{\partial v}{\partial \tilde{t}}+(\boldsymbol{v} \cdot \tilde{\nabla}) v\right]=-\tilde{\nabla} \tilde{p}-\tilde{\rho} \tilde{\nabla} \tilde{\phi}, \\
\frac{\partial}{\partial \tilde{t}}(\tilde{\rho} \tilde{\epsilon})+\tilde{\nabla} \cdot(\tilde{\rho} \tilde{\epsilon} \tilde{v})=\tilde{h}(\tilde{t})(2 \tilde{\rho} \tilde{\epsilon}-3 \tilde{p})-\tilde{p} \tilde{\nabla} \boldsymbol{v}-\tilde{\Lambda},
\end{gathered}
$$

where $\tilde{h}(\tilde{t})=a^{-1}(d a / d \tilde{t})$.

The peculiar gravitational potential $\tilde{\phi}$ is given by the Poisson equation in the present coordinate system as

$$
\tilde{\nabla}^{2} \tilde{\phi}=6 a(\tilde{t})\left(\tilde{\rho}+\tilde{\rho}_{\text {DM }}-1\right),
$$

where $\tilde{\rho}_{\mathrm{DM}}$ is the dimensionless density of the background dark matter and

$$
\tilde{\boldsymbol{\epsilon}}=\frac{1}{\gamma-1} \frac{\tilde{p}}{\tilde{\rho}} .
$$

For the case considered here, $\gamma$ is $5 / 3$ and the first term of the right-hand side of equation (14) vanishes. The term $\pi$ is the dimensionless cooling rate:

$$
\Lambda=\left(a^{7} t_{0} / p_{0}\right) \Lambda .
$$

\section{c) Numerical Hydrodynamics}

We take a cylindrical symmetric coordinate system, i.e., $Z-R$ coordinate, which is adequate for the geometry considered here. The numerical code is constructed following Norman and Winkler (1986), Różyczka (1985), and Hawley, Smarr, and Wilson (1984). The scheme is two-dimensional Eulerian. One time step is divided into two independent steps, i.e., the source and transport steps. In the source step, we accelerate fluid velocities, perform pressure work, and subtract internal energy due to cooling loss. In the transport step, fluid is transported through the computational mesh, for which we employ van Leer's (1977) second-order accurate monotonic interpolation scheme.

In order to handle shock discontinuities, we add a von Neumann-type artificial viscosity in the source step. We adopt the scalar viscosity which behaves like isotropic pressure:

$$
Q=\left\{\begin{array}{cl}
C \rho \cdot(\nabla v)^{2} & \text { for } \nabla \cdot v<0, \\
0 & \text { for } \nabla \cdot v \geq 0 .
\end{array}\right.
$$

Throughout the calculation, we take $C=2.0$. Owing to this viscosity, a shock front is somewhat widely smeared, although this value of $C$ is needed to conserve the total energy.

At the outer boundaries, the following conditions are adopted: $\tilde{\rho}=\tilde{\rho}\left(z_{i}\right), \tilde{p}=\tilde{p}\left(z_{i}\right)$, and $\tilde{v}=0$. On the symmetry axis $(\boldsymbol{R}=0)$, the velocity components perpendicular to the symmetry axis are set to be zero.

We follow the method of Black and Bodenheimer (1975) for calculating the self-gravity. The boundary values of gravitational potential are computed from a multipole expansion. On the symmetry axis $(R=0), \partial \phi / \partial R=0$ should be satisfied. The adopted technique for solving the Poisson equation is the ADI method. Although we try the ILUBCG method, the computational time per step is nearly the same.

In cosmological hydrodynamics, we must consider two types of cooling processes: inverse Compton cooling and radiative cooling. The rate of inverse Compton cooling is given by

$$
\Lambda_{\text {Comp }}=5.4 \times 10^{-36}(1+z)^{4} n \operatorname{ergs~} \mathrm{cm}^{-3} \mathrm{~s}^{-1},
$$

where we took the present $\mathrm{CBR}$ temperature as $2.7 \mathrm{~K}$. The rate of radiative cooling in gas with the primordial abundance $(\mathrm{H}: \mathrm{He}=9: 1$ in number) is approximated by the following functional form (Ikeuchi, Tomisaka, and Ostriker 1983):

$$
\Lambda_{\mathrm{rad}} / n^{2}= \begin{cases}2.4 \times 10^{-27} T^{0.5} & \text { for } T \geq 7 \times 10^{5} \\ 5.9 \times 10^{-16} T^{-1.4} & \text { for } 7 \times 10^{5} \geq T \geq 8 \times 10^{4} \\ 1.9 \times 10^{-42} T^{4.0} & \text { for } 8 \times 10^{4} \geq T \geq 5 \times 10^{4} \\ 8.1 \times 10^{-16} T^{-1.7} & \text { for } 5 \times 10^{4} \geq T \geq 3 \times 10^{4} .\end{cases}
$$

We cut off cooling below $T_{c}=3 \times 10^{4} \mathrm{~K}$, which approximately corresponds to the equilibrium temperature due to UV heating from quasars, some unknown sources, or the blast wave itself. Cooling by molecules such as $\mathrm{H}_{2}$ may make a significant contribution to the total cooling rate at low temperatures if formed (Mac Low and Shull 1986; Shapiro and Kang 1987), but we do not include it.

\section{d) Tests}

We performed several test runs on our hydrodynamical code. They are the Riemann (Sod) shock tube in onedimension, the adiabatic shock wave produced by a point explosion in a stationary, homogeneous medium, and supernova remnants in the interstellar medium in two-dimensional 
$Z-R$ coordinate. Agreements of the results are sufficiently good compared to preexisting analytical, or numerical solutions. The cosmological version of the code is tested in the case of a point explosion in an expanding universe. The adiabatic case without cooling in $\boldsymbol{\Omega}=\boldsymbol{\Omega}_{g}=1$ is compared with the analytical solution (Ikeuchi, Tomisaka, and Ostriker 1983; Bertschinger 1983). The radius of the shock wave obtained by the simulation agrees with the analytical one within one or two mesh sizes; the agreement concerning the density distribution is less sufficient, which may be attributed to the singular nature of the self-similar solution or insufficient resolution of the present calculation. Cases considering cooling in $\Omega=\boldsymbol{\Omega}_{g}=0.2$ and in $\Omega=1$ and $\Omega_{g}=0.2$ are compared with the spherically symmetric hydrodynamical calculation by Vishniac, Ostriker, and Bertschinger (1985). The shock radius agrees within 5\% accuracy. The agreement of the density distributions are fairly good, although the density enhancement behind the shock is relatively small because of the resolution. Conservations of mass and momentum are automatically achieved by the numerical scheme. Total energy conserves within the error of $3 \%-5 \%$

\section{RESULTS}

\section{a) Initial Condition}

Initially ambient gas is at rest in comoving coordinates and has density $\rho_{g}=\Omega_{g} \rho_{\text {crit }}$. The temperature of the ambient gas is assumed to be $T_{i}=100 \mathrm{~K}$. This choice does not have any reasoning, but it will not affect the results as long as $T_{i}$ is as small as this order, since a sufficiently developed, cosmological blast wave is pushed by gravity, not by pressure.

As seen in Figure 1, the first explosion with the energy $E_{i, 1}$ is set at the redshift $z_{i, 1}$ and the second explosion with the energy $E_{i, 2}$ at the redshift $z_{i, 2}$ at the comoving distance $d$. Throughout the calculations, we take $E_{i, 1}=E_{i, 2} \equiv E_{i}$. In the first, we set the explosion redshifts to be $z_{i, 1}=z^{i, 2}$, and then we also examine the cases of different $z_{i}$. Energy of each explosion is deposited as thermal energy into several mesh volumes.

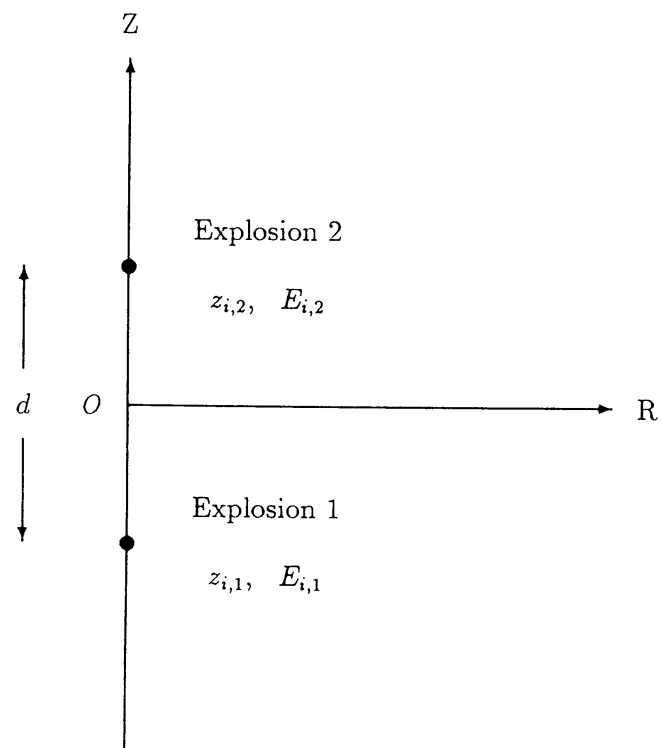

FIG. 1.-Configuration of the present calculation. The first explosion with energy $E_{i, 1}$ occurs at the redshift $z_{i, 1}$ and the second explosion with energy $E_{i, 2}$ occurs at the redshift $z_{i, 2}$. The two explosion points are put at the comoving distance $d$ Mpc.

\section{b) Results for Same Explosion Redshifts}

We summarize parameters of various models in Table 1.

The results of calculations can be classified into four types by the appearances of global structures: (1) merging case (M case), (2) merging case with a ring (MR case), (3) nonmerging case with a ring (WR case), and (4) nonmerging case with a wall (W case). We present typical examples of these four cases for

TABLE $1 \mathrm{~A}$

NUMERICAL ResUlts FOR THE HD MODEL $\left(\Omega=1, \Omega_{g}=1, E_{i}=10^{61}\right.$ ergs, $\left.\Delta=0.06 \mathrm{Mpc}\right)$

\begin{tabular}{cccccccc}
\hline$z_{i}$ & $d$ & Merge & $V_{\text {sw }}$ & $V_{\text {sw }} / V_{\mathbf{0}}$ & $R_{\text {sw }}$ & Ring & $\kappa_{\text {max }}$ \\
\hline $5 \ldots \ldots$ & 1.2 & Yes & 94.8 & 1.54 & 2.83 & No & $\ldots$ \\
& 1.8 & Yes & 99.0 & 1.61 & 2.87 & No & $\ldots$ \\
& 2.4 & Yes & 104.4 & 1.69 & 2.92 & Yes & 18.0 \\
& 3.0 & Yes & 111.2 & 1.81 & 2.98 & Yes & 21.5 \\
$10 \ldots \ldots$ & 3.6 & No & $\ldots$ & $\ldots$ & $\ldots$ & No & $\ldots$ \\
& 1.2 & Yes & 85.6 & 1.55 & 2.73 & No & $\ldots$ \\
& 1.8 & Yes & 89.8 & 1.63 & 2.78 & Yes & 20.5 \\
& 2.4 & Yes & 95.3 & 1.73 & 2.83 & Yes & 28.5 \\
& 3.0 & No & $\ldots$ & $\ldots$ & $\ldots$ & Yes & 19.7 \\
& 3.6 & No & $\ldots$ & $\ldots$ & $\ldots$ & No & $\ldots$ \\
& 1.2 & Yes & 62.2 & 1.56 & 2.46 & Yes & 18.7 \\
& 1.8 & Yes & 66.4 & 1.66 & 2.51 & Yes & 22.8 \\
& 2.4 & No & $\ldots$ & $\ldots$ & $\ldots$ & Yes & 25.0 \\
& 3.0 & No & $\ldots$ & $\ldots$ & $\ldots$ & Yes & 12.6 \\
& 3.6 & No & $\ldots$ & $\ldots$ & $\ldots$ & No & $\ldots$ \\
& 1.2 & Yes & 48.6 & 1.57 & 2.26 & Yes & 19.2 \\
& 1.8 & Yes & 52.3 & 1.69 & 2.32 & Yes & 19.3 \\
& 2.4 & No & $\ldots$ & $\ldots$ & $\ldots$ & Yes & 17.4 \\
& 3.0 & No & $\ldots$ & $\ldots$ & $\ldots$ & No & $\ldots$ \\
\hline
\end{tabular}

TABLE 1B

NUMERICAL RESULTS FOR THE LD MODEL $\left(\Omega=0.1, \Omega_{g}=0.1, E_{i}=10^{61} \mathrm{ergs}, \Delta=0.1 \mathrm{Mpc}\right)$

\begin{tabular}{cccccccc}
\hline \hline$z_{i}$ & $d$ & Merge & $V_{\text {sw }}$ & $V_{\text {sw }} / V_{\mathbf{o}}$ & $R_{\text {sw }}$ & Ring & $\kappa_{\text {max }}$ \\
\hline $10 \ldots \ldots$ & 2.0 & Yes & 432.1 & 1.53 & 4.69 & No & $\ldots$ \\
& 3.0 & Yes & 447.9 & 1.59 & 4.75 & No & $\ldots$ \\
& 4.0 & Yes & 477.3 & 1.70 & 4.85 & Yes & 9.0 \\
& 5.0 & Yes & 509.9 & 1.81 & 4.97 & Yes & 12.1 \\
$20 \ldots \ldots$ & 6.0 & No & $\ldots$ & $\ldots$ & $\ldots$ & Yes & 10.6 \\
& 2.0 & Yes & 252.9 & 1.52 & 3.92 & Yes & 12.0 \\
& 3.0 & Yes & 273.5 & 1.64 & 4.03 & Yes & 21.4 \\
& 4.0 & No & $\ldots$ & $\ldots$ & $\ldots$ & Yes & 18.3 \\
$30 \ldots$. & 5.0 & No & $\ldots$ & $\ldots$ & $\ldots$ & No & $\ldots$ \\
& 1.0 & Yes & 181.4 & 1.45 & 3.51 & No & $\ldots$ \\
& 2.0 & Yes & 190.7 & 1.52 & 3.57 & Yes & 15.8 \\
& 3.0 & Yes & 211.1 & 1.69 & 3.69 & Yes & 20.8 \\
& 4.0 & No & $\ldots$ & $\ldots$ & $\ldots$ & Yes & 14.3 \\
\hline
\end{tabular}

TABLE 1C

NUMERICAL RESULTS FOR THE DM MODEL $\left(\Omega=1, \Omega_{g}=0.1, E_{i}=10^{61} \mathrm{ergs}, \Delta=0.1 \mathrm{Mpc}\right)$

\begin{tabular}{cccccccc}
\hline$z_{i}$ & $d$ & Merge & $V_{\text {sw }}$ & $V_{\text {sw }} / Y_{0}$ & $R_{\text {sw }}$ & Ring & $\kappa_{\text {max }}$ \\
\hline $10 \ldots \ldots$ & 2.0 & Yes & 256.1 & 1.56 & 3.94 & No & $\ldots$ \\
& 3.0 & Yes & 271.0 & 1.65 & 4.01 & No & $\ldots$ \\
& 4.0 & Yes & 293.2 & 1.79 & 4.12 & No & $\ldots$ \\
& 5.0 & Yes & 312.7 & 1.90 & 4.21 & No & $\ldots$ \\
$20 \ldots \ldots$ & 6.0 & No & $\ldots$ & $\ldots$ & $\ldots$ & No & $\ldots$ \\
& 2.0 & Yes & 124.1 & 1.60 & 3.09 & No & $\ldots$ \\
$30 \ldots \ldots$ & 3.0 & Yes & 137.6 & 1.78 & 3.20 & Yes & 7.8 \\
& 4.0 & No & $\ldots$ & $\ldots$ & $\ldots$ & No & $\ldots$ \\
& 1.0 & Yes & 71.9 & 1.49 & 2.58 & No & $\ldots$ \\
& 2.0 & No & $\ldots$ & $\ldots$ & $\ldots$ & Yes & 9.6 \\
& 3.0 & No & $\ldots$ & $\ldots$ & $\ldots$ & No & $\ldots$ \\
\hline
\end{tabular}


the model universe $\Omega=\Omega_{g}=1\left(z_{i, 1}=z_{i, 2}=z_{i}\right.$, and $E_{i}=10^{61}$ ergs). These evolutionary features are shown in Figures 2-7. All of contour maps of density, pressure, etc., are shown in terms of the dimensionless values. Arrows in the contour maps represent peculiar velocity fields.

\section{i) Merging Case $\left(z_{i}=10\right.$, and $\left.d=1.2 \mathrm{Mpc}\right)$ : Model $A$}

The evolution of the density distribution in this case is illustrated in Figure 2. Shock waves produced by explosions at the same redshift $z_{\mathrm{i}}=10$ collide at $z \approx 9.5$, when the shock waves have not been yet cooled by Compton cooling. Then, the collision occurs during the adiabatic phase. Immediately after the collision, reflected shocks emerge from at the plane of interaction and move outward perpendicular to the plane of the interaction. This situation is clearly seen in Figure $2 b$ at the epoch $z=7.2$. We can see the positions of reflected shocks by steep gradients of density contours or velocity fields. After the reflected shocks overtake the outer shocks, disordered gas motions arise due to the disturbing waves in the cavity, and eventually the two outer shocks merge into a single shell. The final structure can be hardly distinguished from that produced by a single explosion, except that it is somewhat elongated along the symmetry axis.

ii) Merging Case with a Ring $\left(z_{i}=10\right.$, and $\left.d=2.4 \mathrm{Mpc}\right)$ : Model $B$

In this case, the explosion redshift is the same as that of the model A, but the distance between the two explosions is twice as large. The evolution is illustrated in Figure 3. Although the shock waves do not suffer significant cooling before the collision, the cooled dense shells begin to form soon after the collision, and weak reflected shocks emerge. The weak reflected shocks disappear in a short time, and the outer shock waves merge into a single shell by $z=0$ as is seen in Figure $3 d$. The clearly different feature from model $\mathrm{A}$ is the formation of a high-density region at the spot where the two outer shocks are colliding. This high-density region is a ring from the geometry. The density enhancement of the ring is $\kappa=\rho_{\text {ring }} / \rho_{0}=28.5$, which is larger than that of the outer shell. The mass of the ring is $M_{r} \sim 3 \times 10^{12} M_{\odot}$.

iii) Nonmerging Case with a Ring $\left(z_{i}=20\right.$, and $\left.d=2.4 \mathrm{Mpc}\right)$ : Model C

In this case (Fig. 4), the explosion redshift $z_{i}=20$ far earlier than model B. At such a high redshift, the inverse Compton cooling is so efficient that cooled dense shells are formed before the collision. At the collision time, the shells have been completely cooled, and reflected shocks do not emerge. Instead, a dense wall is formed at the plane of the interaction. Similarly to model B, a high-density ring is formed as seen in Figure $4 d$. The density enhancement of the ring is $\kappa=25.0$, and its mass is $\sim 10^{12} M_{\odot}$.

For this case, the calculation with finer mesh size $\Delta=0.03$ $\mathrm{Mpc}$, which is half as large as in the other calculations, is also performed (Fig. 5). The global structure, the radius of the shock wave, and the major radius of the ring are almost the same as those with the ordinary mesh size of $\Delta=0.06 \mathrm{Mpc}$. On the other hand, the shell thickness changes from $\approx 0.3 \mathrm{Mpc}$ for $\Delta=0.06 \mathrm{Mpc}$ to $\approx 0.2 \mathrm{Mpc}$ for $\Delta=0.03 \mathrm{Mpc}$; the minor diameter of the ring changes from $\approx 0.3 \mathrm{Mpc}$ for $\Delta=0.06 \mathrm{Mpc}$ to $0.2 \mathrm{Mpc}$ for $\Delta=0.03 \mathrm{Mpc}$; the density enhancement of the ring is $\kappa=52.9$, which is about twice as large as that of the low-resolution calculations. This result suggests to us that the calculations with $\Delta=0.06 \mathrm{Mpc}$ cannot resolve the fine structure of the ring, but they can reproduce the same evolution and global structures. Closed-up views at $Z=0$ near the plane of the interaction and the ring are shown in Figure 6. Figures $6 a$ and $6 b$ show the density and pressure contours in addition to the velocity fields, respectively. The ring grows gathering
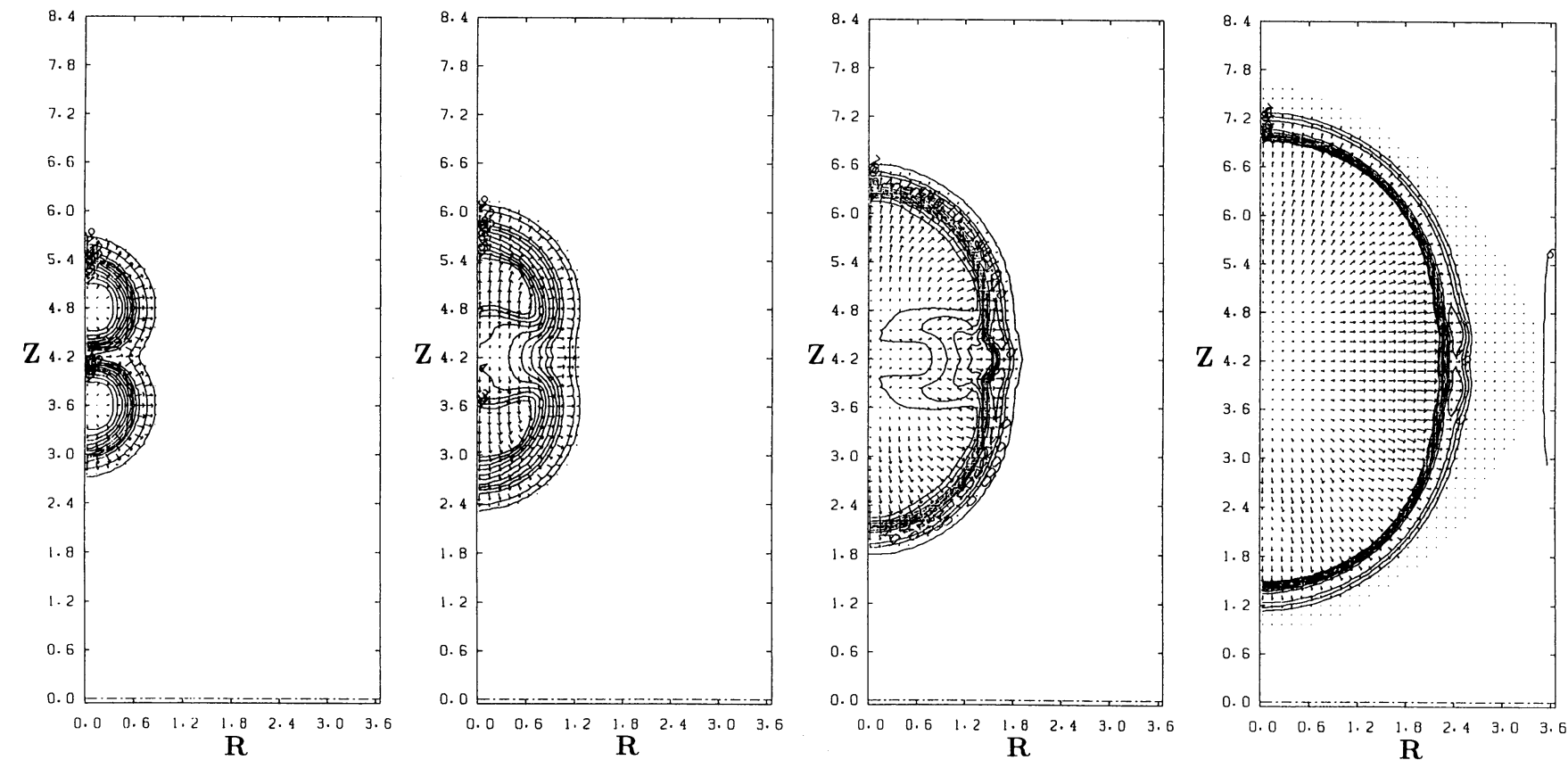

Fig. 2.-Evolution of the density distribution for model $\mathrm{A}\left(\Omega=1, \Omega_{g}=1, z_{i}=10, E_{i}=10^{61} \mathrm{ergs}\right.$, and $\left.d=1.2 \mathrm{Mpc}\right)$, representing the complete merging case. The mesh size is $\Delta=0.06 \mathrm{Mpc}$. The redshifts are $9.1,7.2,3.4$, and 0.0 from left to right. The density is shown by the dimensionless value, normalized by the average density of the universe. Each contour interval is a factor of $10^{2 / 11}$, and only the contours of the top 10 levels are plotted. The logarithms of the maximum levels of the density contours are $0.204,0.252,0.557$, and 0.716 for each epoch. The peculiar velocity fields are also shown by the arrows, whose lengths are proportional to the absolute values of velocities. The maximum velocities are $8.3 \times 10^{2} \mathrm{~km} \mathrm{~s}^{-1}, 4.6 \times 10^{2} \mathrm{~km} \mathrm{~s}^{-1}, 2.0 \times 10^{2} \mathrm{~km} \mathrm{~s}^{-1}$, and $1.1 \times 10^{2} \mathrm{~km} \mathrm{~s}^{-1}$, respectively. 


$$
\frac{3333}{3333}
$$




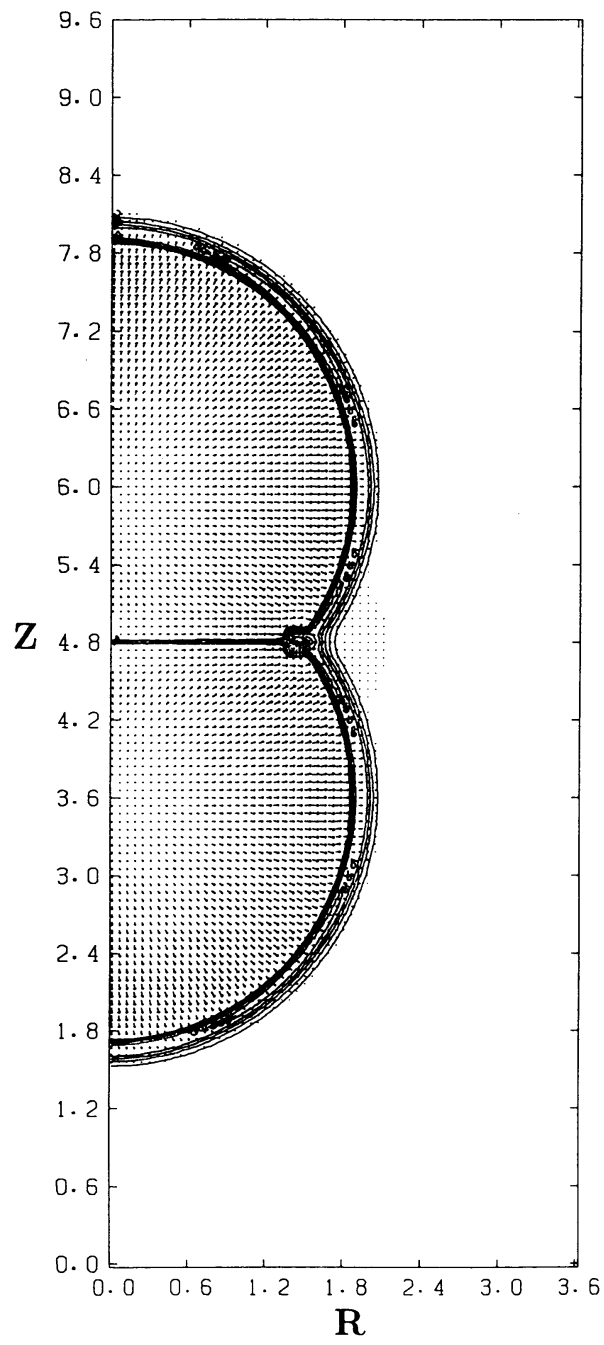

Fig. 5.-High-resolution calculation with mesh size $\Delta=0.03$ Mpc. Parameters are the same as in Fig. 4. This shows the density contour with the velocity field at $z=0$. The logarithm of the maximum level of the density contour is 1.527. The maximum velocity is $1.1 \times 10^{2} \mathrm{~km} \mathrm{~s}^{-1}$.

matter from the cooled shells and the wall. Figures $6 c$ and $6 d$ illustrate the contours of the peculiar potential $\phi$. At the ring, a deep potential well is formed, but this does not play a role in accumulating matter to the ring, as is seen from the arrows of the velocity fields. The physical cause of ring formation is discussed in $\S$ IV.

iv) Nonmerging Case $\left(z_{i}=20\right.$, and $\left.d=3.6 \mathrm{Mpc}\right)$ : Model D

The distance between two explosions is the largest of the cases explored. The collision just occurs at the epoch $z \approx 3$, when cooled, dense shells have already been formed. In this case, a relatively dense wall is formed, but no ring feature appears (Fig. 7), because there is no time needed to form a ring. This reason is also discussed in $\S \mathrm{IV}$.

\section{c) Parameter Dependence}

Here, we survey the parameter dependence of the global structure produced by the collision of shock waves. The objects of the calculation are to see under what conditions two shocks are merged into a single shell and whether they form a ring by the present $z=0$. i) Dependence on the Explosion Redshift and the Separation of the Two Explosion Points

First, we examine the dependence on the explosion redshift $z_{i}$ and the separation of the two explosions $d$. The explosion energy is set to $E_{i}=10^{61}$ ergs. In the case of the HD model $\left(\Omega=\Omega_{g}=1\right)$, the dependence of the structure formed on $z_{i}$ and $d$ is summarized in Figure $8 a$. At a fixed explosion redshift (e.g., $z_{i}=20$ ), the structures change according to the distance $d$ as follows. For a fairly small $d$, the two shock waves completely merge into a single shell, not forming a ring. With increasing $d$, the two shock waves merge into a single shell, forming a highdensity ring. With further increasing $d$, the two shock waves do not merge and leaving a wall structure in the plane as well as the ringlike density enhancement. For a still larger $d$, the shock waves do not form a ring. The critical distances classifying the above four cases decrease with increasing explosion redshift $z_{i}$. Since at a larger redshift the Compton cooling is efficient, cooled dense shells are quickly formed, and they can merge only for smaller $d$. Strong cooling at large redshifts prohibits a shock wave expanding to a larger size, and thus the critical distances scale to smaller ones.

The LD model $\left(\Omega=\Omega_{g}=0.1\right)$ and the DM model $(\Omega=1$, and $\Omega_{g}=0.1$ ) show similar parameter dependences as shown in Figures $8 b$ and $8 c$, respectively, although the classification of structures formed cannot be made so definitely in the latter model. These parameter dependences are discussed in relation to the physical cause of ring formation in $\S$ IV.

ii) Dependence on the Explosion Energy

Since a larger explosion energy makes a larger shell, the increase in the explosion energy leaving $d$ unchanged will have a similar effect to the case of decreasing $d$ leaving $E_{i}$ unchanged. Here, we examine the dependence on the explosion energy using the scale transformation. As given in $\S$ II, all physical quantities are transformed to nondimensional form by dividing zero-subscripted constants such as $r_{0}, t_{0}$, and $\rho_{0}$. These constants scale with respect to the scale transformation $r_{0} \rightarrow \beta r_{0}$ as follows:

$$
\begin{array}{cl}
t_{0} \rightarrow t_{0}, & \rho_{0} \rightarrow \rho_{0}, \quad v_{0} \rightarrow \beta v_{0}, \quad p_{0} \rightarrow \beta^{2} p_{0}, \\
\epsilon_{0} \rightarrow \beta^{2} \epsilon_{0}, \quad \phi_{0} \rightarrow \beta^{2} \phi_{0} .
\end{array}
$$

The total energy scales as $E \rightarrow \beta^{5} E$. Therefore, models in which $r_{0} \rightarrow \beta r_{0}$, and $E_{i} \rightarrow \beta^{5} E_{i}$ are all similar. This similarity holds perfectly only for the adiabatic phase in which any cooling process is not effective. On the other hand, for the radiative phase cooling is effectively more efficient in the dimensionless forms in the case of larger $E_{i}$, i.e., larger $r_{0}$. Utilizing this scaling law, we can easily see the essential effect of changing the explosion energy.

We calculated two models in addition to model $\mathrm{C}(\boldsymbol{\Omega}=$ $\Omega_{g}=1, z_{i}=20, E_{i}=10^{61} \mathrm{ergs}$, and $d=2.4 \mathrm{Mpc}$ ), the model $\mathrm{E}$ for $E_{i}=10^{59} \mathrm{ergs}$, and $d=0.96 \mathrm{Mpc}=2.4 \times 100^{-1 / 5} \mathrm{Mpc}$, and the model $\mathrm{F}$ for $E_{i}=10^{63}$ ergs, and $d=6$ $\mathrm{Mpc}=2.4 \times 100^{1 / 5} \mathrm{Mpc}$. Density contour maps at $z=0$ of models $\mathrm{E}$ and $\mathrm{F}$ are shown, respectively, in Figures $9 a$ and $9 b$, which should be compared with Figure $4 d$ for model $\mathrm{C}$. The global appearances are very similar: a wall as well as a dense ring is formed. At the range of $E_{i}=10^{59}-10^{63} \mathrm{ergs}$, the above scaling law holds within the error of $\sim 1 \%$ for the shock radius, of $\sim 5 \%$ for the ring major radius, and of $\sim 5 \%$ for the total energy. The density enhancements in the rings show the energy dependence as $\kappa=\rho_{\text {ring }} / \rho_{0}=25\left(E_{i} / 10^{61} \mathrm{ergs}\right)^{0.1}$. 


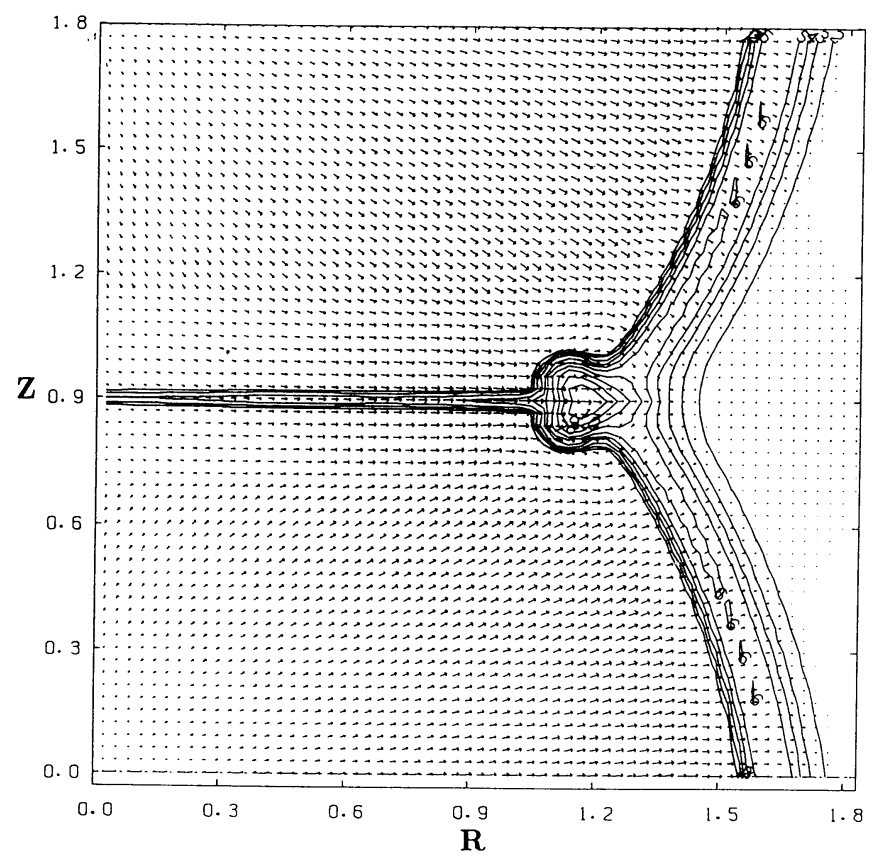

FIG. $6 a$

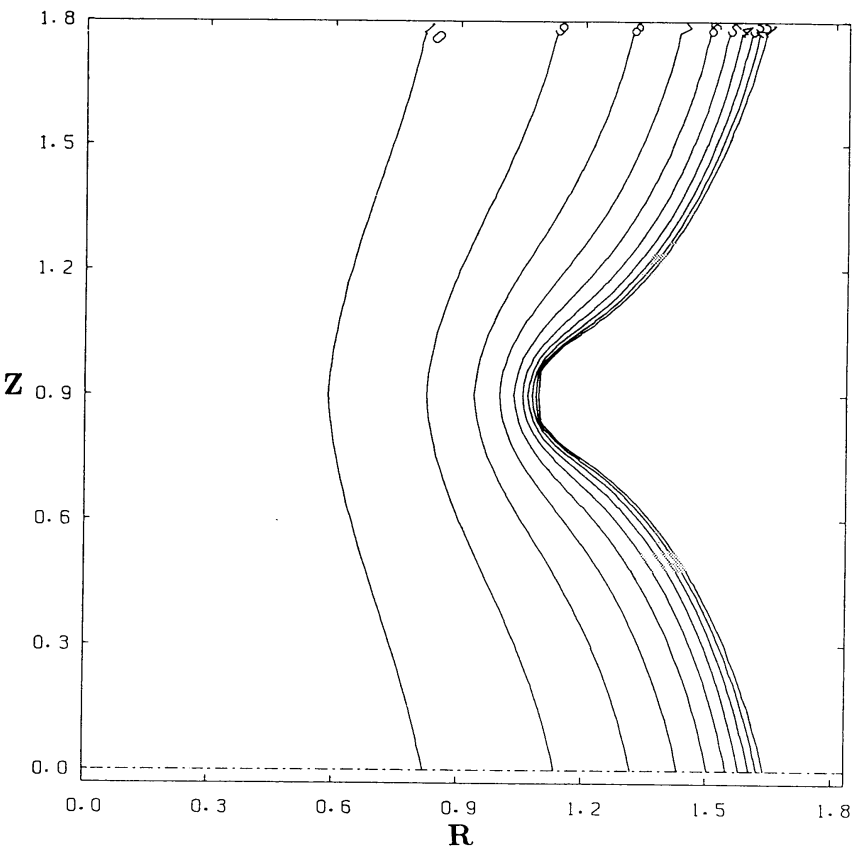

FIG. $6 c$

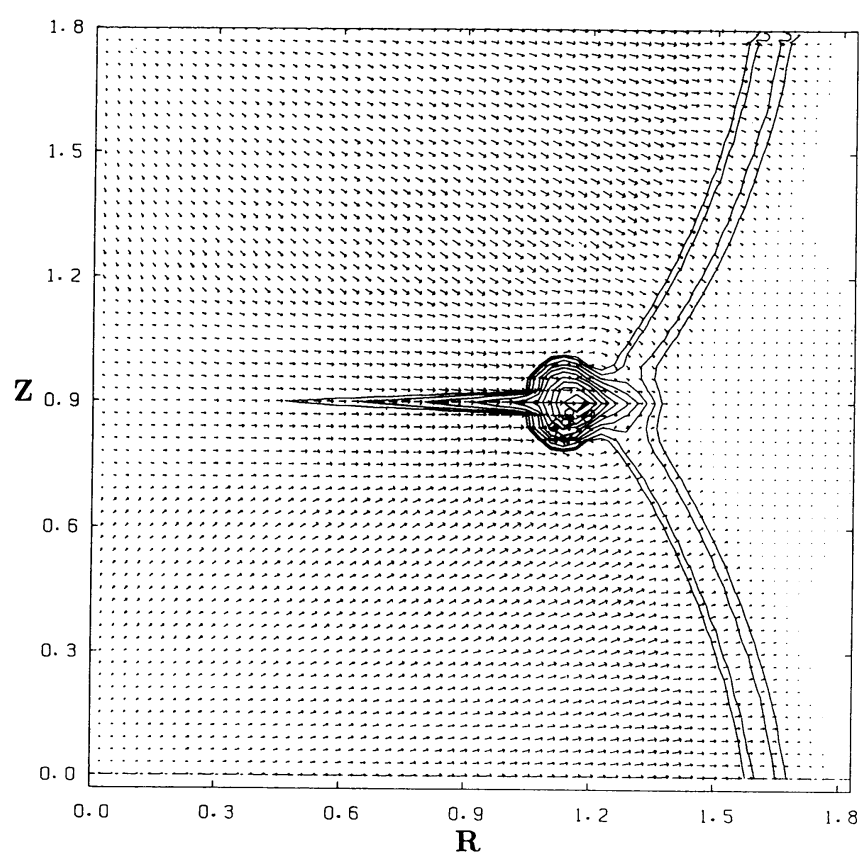

FIG. $6 b$

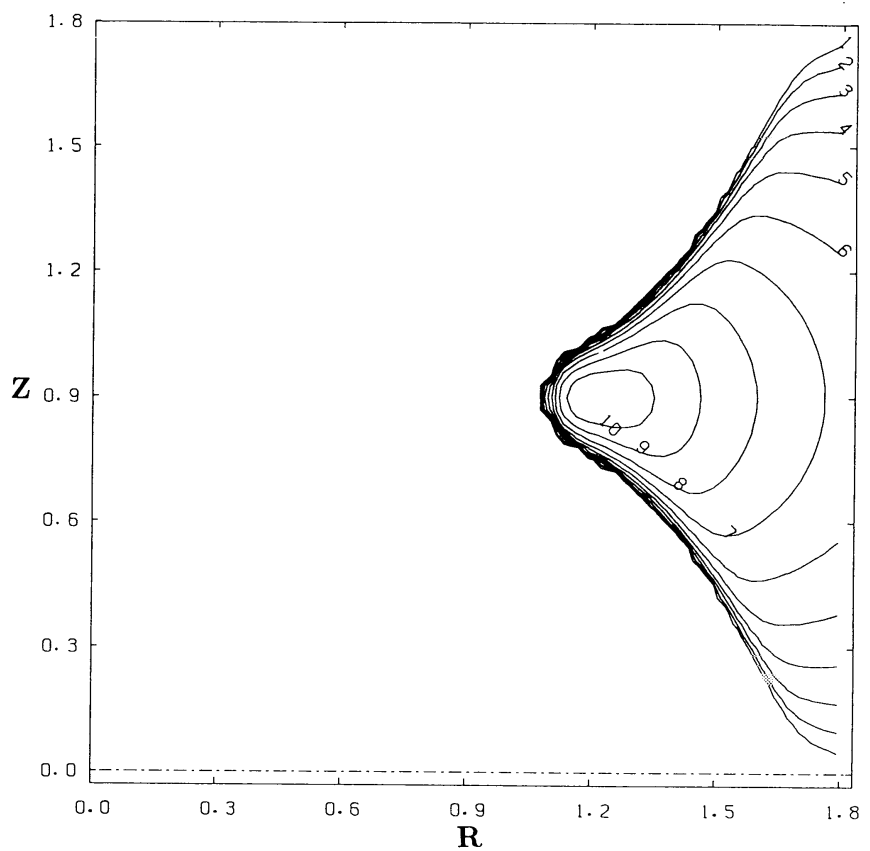

FIG. $6 d$

FIG. 6.-Closed-up views of the high-resolution calculation. (a) Contour maps of the distribution of dimensionless density. The logarithms of the maximum level

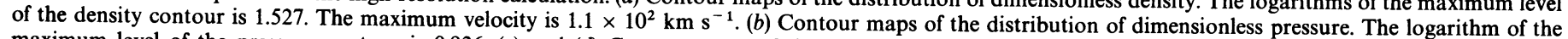

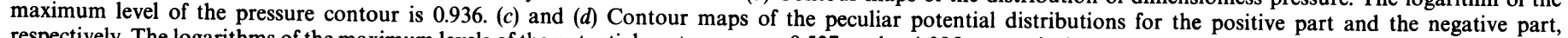
respectively. The logarithms of the maximum levels of the potential contours are -0.537 , and -1.285 , respectively.

\section{d) Results for Different Explosion Redshifts}

Here, we will present two examples of the case that two explosions occur at different epochs. In the model G (Fig. 10), the first explosion occurs at $z_{i, 1}=20$, and the second explosion occurs at $z_{i, 2}=10$, when the first one has already formed a cooled, dense shell (Fig. 10a). Both explosion energies are set $E_{i}=10^{61}$ ergs. The collision of the two shock waves occurs at $z \sim 8$, at which both shocks are in the radiative stage. After the collision, a wall forms, but by $z=0$ it almost disappears accompanying a dense ring. The density enhancement of the ring is $\kappa=21.7$, which is somewhat smaller than the corresponding cases with simultaneous explosions: $\kappa=25.0$ for $z_{i, 1}=z_{i, 2}=20$, and $\kappa=28.5$, for $z_{i, 1}=z_{i, 2}=10$.

In the model $\mathbf{H}$ (Fig. 11), the first explosion occurs at $z_{i, 1}=$ 20 and the second one occurs at $z_{i, 2}=5$. At the time of the 

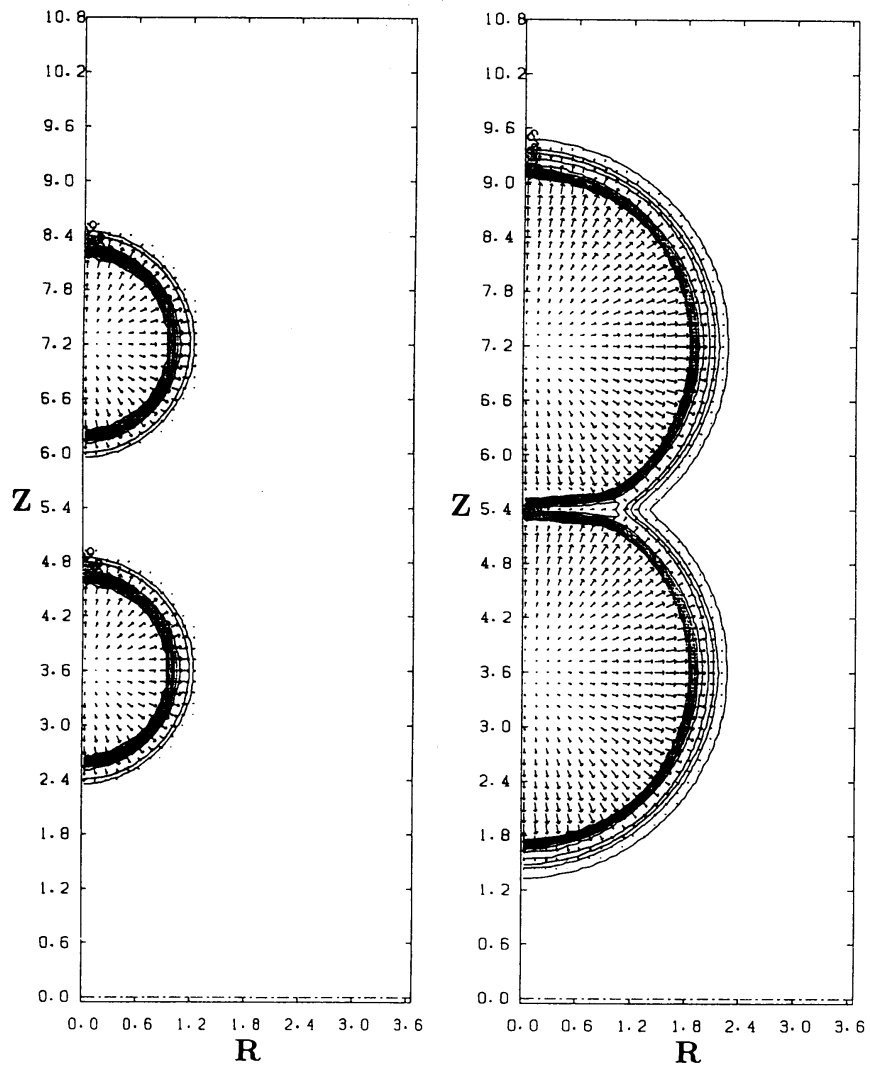

Fig. 7.-Evolution of the density distribution for model $\mathrm{D}\left(\boldsymbol{\Omega}=1, \Omega_{g}=1\right.$, $z_{i}=20, E_{i}=10^{61} \mathrm{ergs}$, and $d=3.6 \mathrm{Mpc}$ ) representing the no-merging case. The redshifts are 10.1 and 0.0 from left to right. The logarithms of the maximum levels of the density contours are 0.338 and 0.736 , respectively. The maximum velocities are $2.7 \times 10^{2} \mathrm{~km} \mathrm{~s}^{-1}$, and $8.7 \times 10^{1} \mathrm{~km} \mathrm{~s}^{-1}$, respectively.

collision the first explosion has formed a cooled, dense shell, but the second one does not suffer sufficient cooling, remaining in the adiabatic phase. The high-pressure gas in the second shock can break the dense shell of the first shock, and a strong shock wave goes into the cavity of the first shell, as is seen in a rapid change of velocity vectors in Figure $11 \mathrm{c}$. At $z=0$, the two shocks merge into a single shell, leaving only a trailing structure which is the debris of the dense shell of the first explosion. The maximum value of the density enhancement in the ringlike region $\kappa=13.0$.

From the above calculations, we can conclude that the younger shock works to inhibit the formation of a wall or a ring, although a dense ring can be produced when the younger shock wave has already suffered much cooling before the collision.

\section{PHYSICAL ANALYSIS OF NUMERICAL RESULTS}

\section{a) Physical Cause of Ring Formation}

As seen in $\S$ III, self-gravity is not the main cause for the ring formation, but the hydrodynamical interaction of colliding gas leads to the ring formation. This statement can be justified by the fact that the shock collision in non-self-gravitating interstellar medium, which was calculated as a test, results in ring formation in agreement with the result by Różyczka et al. Suppose that two cooled, dense shells produced by two explosions with the same energy collide at the plane of the interaction. When the collision of matter is perfectly head-on, the matter loses its momentum normal to the plane and stops in the plane. When the collision of matter occurs obliquely, its component of momentum perpendicular to the plane is lost, but the parallel component is conserved. Since the shock waves decelerate as time goes on, the matter moves along the plane to overtake the shock front. A ringlike density enhancement is formed at the place where the two outer shock waves intersect. Ring formation does not occur when radiative cooling is ineffective, because in such a case reflected shock waves expand inside the cavity as the incident angle of the shock waves increases, and gas with zero momentum in perpendicular to the plane does not remain along the plane.

Here, we will analyze this process in some detail. For simplicity, we first examine the collision of two identical spherical shocks in a nonexpanding, nongravitating medium. The expansion law of the shock wave is supposed to follow a power law, $R_{s}=C t^{\alpha}$. We assume the shock waves are completely cooled and the pressure does not affect the dynamics. We follow the trajectory of a mass particle $P$. The configuration is shown in Figure 12. The mass particle $P$ starting from point $P_{i}$, whose distance from the explosion point $Q$ is $r_{p}$, begins to be entrained into the shocked shell at the time $t_{s}$, where $t_{s}$ is given by $r_{p}=R_{s}\left(t_{s}\right)$. The particle $P$ collides with another shell at the time $t_{c}$, where $t_{c}$ is given by

$$
R_{s}\left(t_{c}\right)=C t_{c}^{\alpha}=l \sec \theta(=d \sec \theta / 2),
$$

where $\theta$ is the angle $O Q P_{i}$ and $l$ is the distance from $Q$ to the interaction plane. Thus, we have

$$
t_{c}=(l \sec \theta / C)^{1 / \alpha} .
$$

The particle $P$ loses its momentum perpendicular to the plane and moves along the plane conserving its momentum parallel to the plane at $t>t_{c}$. If it is a free particle, it will move at the constant speed

$$
v_{p}=v_{s}\left(t_{c}\right) \sin \theta=\alpha C t_{c}^{\alpha-1} \sin \theta .
$$

Therefore, the position of the particle $P$ at time $t$ is given by

$$
\begin{aligned}
& q_{p}=l \tan \theta+v_{p}\left(t-t_{c}\right)=l \tan \theta \\
& +\alpha C(l \sec \theta / C)^{(\alpha-1) / \alpha} \sin \theta\left(t-t_{c}\right) .
\end{aligned}
$$

On the other hand, the position of the shocked shell along the plane is given by

$$
q_{s}=\left(R_{s}^{2}-l^{2}\right)^{1 / 2}=\left(C^{2} t^{2 \alpha}-l^{2}\right)^{1 / 2},
$$

and its velocity is

$$
\dot{q}_{s}=\frac{\alpha C^{2} t^{2 \alpha-1}}{\left(C^{2} t^{2 \alpha}-l^{2}\right)^{1 / 2}} .
$$

Then, for $R_{s}=C t^{\alpha} \gg l$, and if $\alpha<1$, the shock wave decelerates and the particle $P$ with the constant speed will catch up to it soon after.

Figure $13 a$ shows the trajectories of colliding particles in the case of the momentum-driven snowplow phase in the stationary medium, i.e., $\alpha=\frac{1}{4}$. The time $t$ measured from the explosion time is normalized by $t_{\text {coll }}$ which is defined by $2 R_{s}\left(t_{\mathrm{coll}}\right)=$ $d=2 l$. The distance $q$ along the symmetry plane from the origin is normalized by $l=d / 2$. The solid lines show the trajectories of mass particles, corresponding to the angle $\theta$ from $10^{\circ}$ to $60^{\circ}$ in every $5^{\circ}$ from below. The dashed line indicates the trajectory of the shock intersection. The trajectories of mass particles of $\theta \sim 35^{\circ}-50^{\circ}$ converge to nearly the same spot $q_{p} \approx$ 1.6 at $t \approx 13$, where a dense ring is to be formed. Soon after, the 


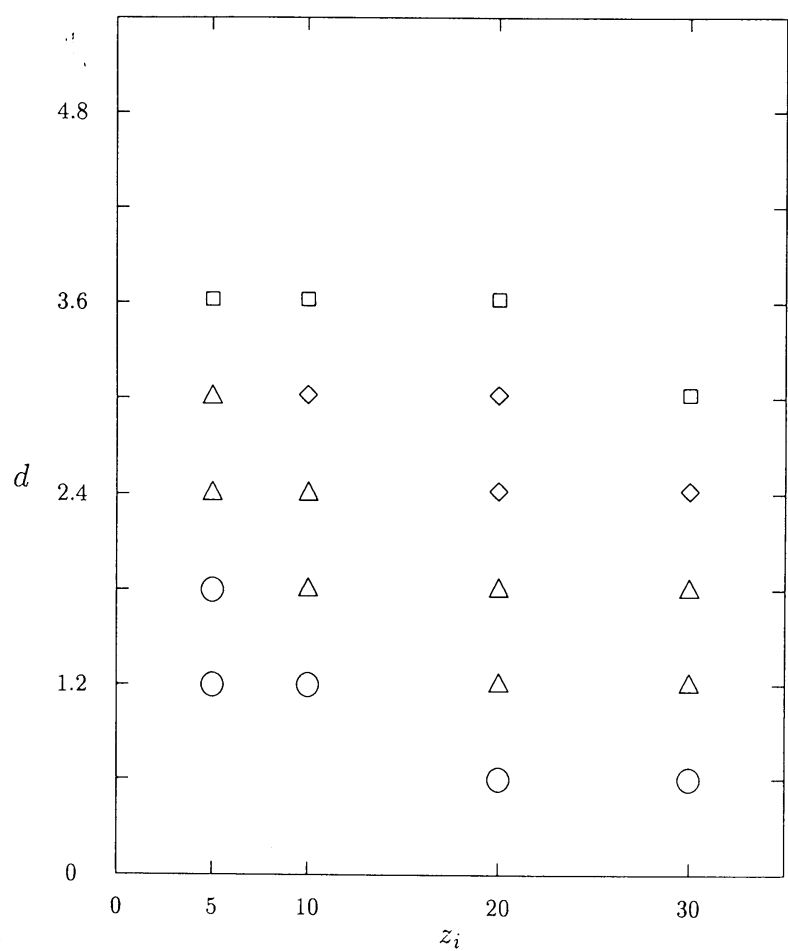

FIG. $8 a$

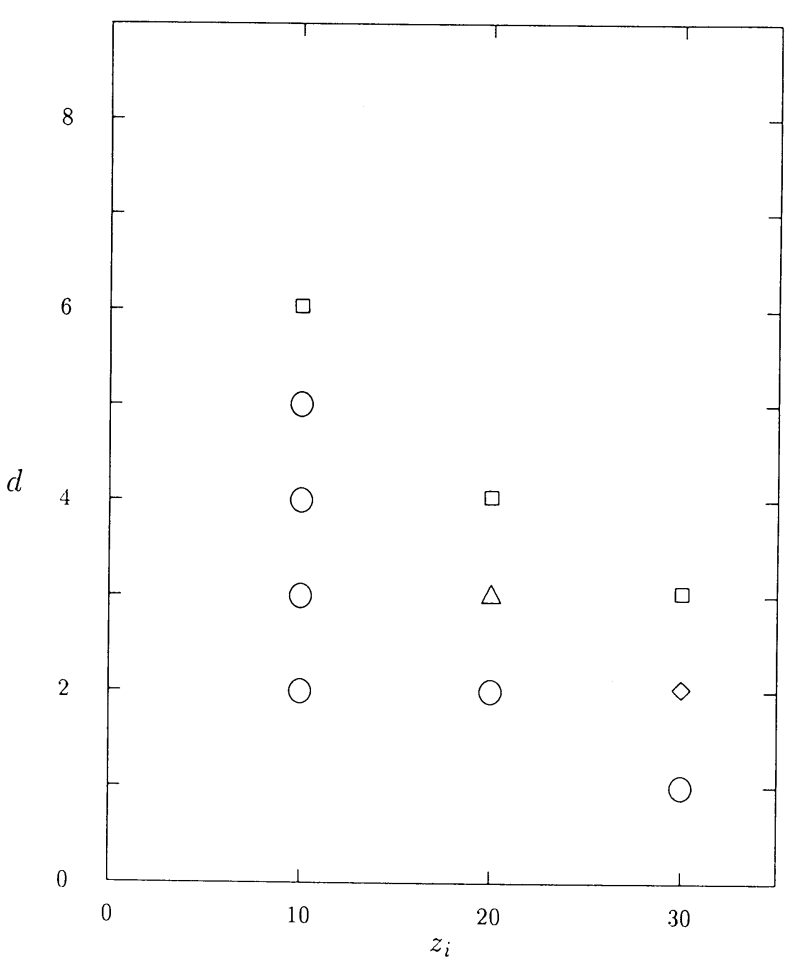

Fig. $8 c$

Fig. 8.-Classification of produced structures. (a) The case of the HD model $\left(\Omega=1, \Omega_{g}=1\right)$, and $E_{i}=10^{61} \mathrm{ergs}$. Open circles, triangles, diamonds, and squares represent the merging cases, merging cases with a ring, nomerging cases with a ring, and no-merging cases, respectively. All calculations were perfomed with $\Delta=0.06 \mathrm{Mpc}$. (b) The case of the LD model $(\Omega=0.1$, $\Omega_{g}=0.1$, and $E_{i}=10^{61}$ ergs. All calculations were performed with $\Delta=0.1$ Mpc. Each symbol has the same meaning as in $(a)$. (c) The case of the DM model $\left(\Omega=1, \Omega_{g}=0.1\right)$, and $E_{i}=10^{61}$ ergs. All calculations were performed with $\Delta=0.1 \mathrm{Mpc}$. Each symbol has the same meaning as in $(a)$.

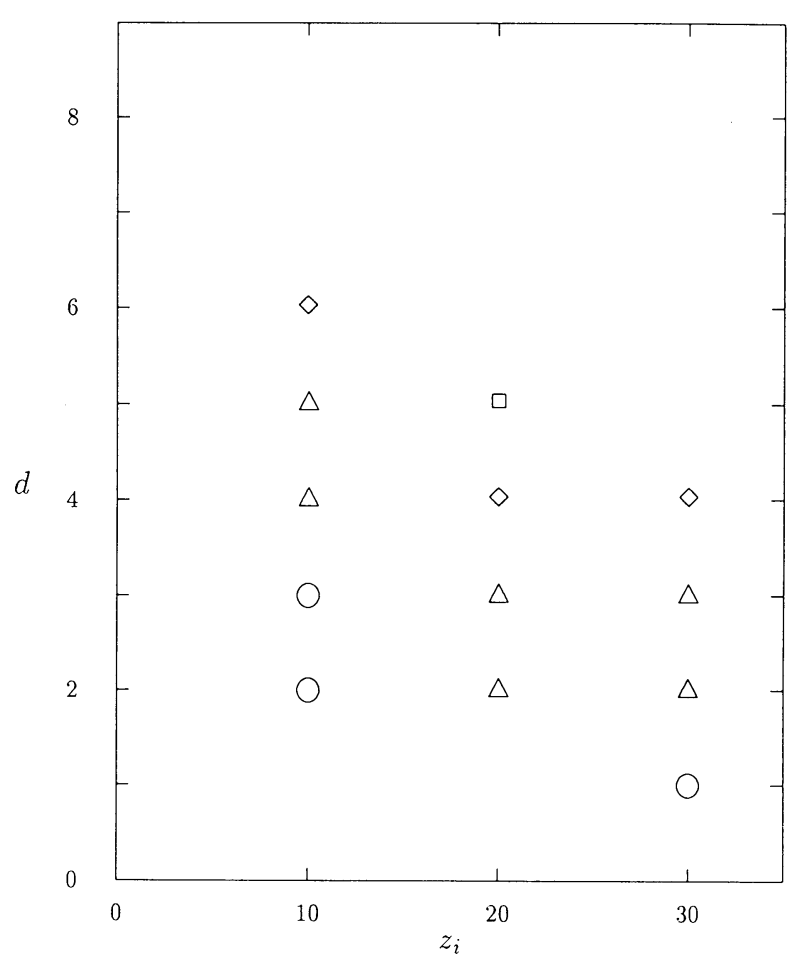

FIG. $8 b$

convergent point catches up to the intersection point of the outer shock waves and the ring grows with collecting matter.

We can make a similar analysis in an expanding medium. Figure $13 b$ shows the case of the cosmological adiabatic blast wave with index $\alpha=\frac{4}{5}$. The time $t$ measured from the explosion time is normalized by $t_{\text {coll }}$ which is defined by $2 R_{s}\left(t_{\text {coll }}\right)$ $\left(1+z_{\text {coll }}\right)=d=2 l$. The comoving distance $q$ is normalized by $l=d / 2$. In this figure, the solid lines show the trajectories of mass particles corresponding to the angle $\theta$ from $20^{\circ}$ to $50^{\circ}$ in every $5^{\circ}$ from below. The dashed line indicates the position of the shock intersection. In this case, the particles with $\theta \sim 35^{\circ}-$ $50^{\circ}$ converge to nearly the same spot $q_{p} \approx 1.3$ at $t \approx 50$. Hence, we may conclude that the ring can be formed after the intersection point of shock waves reaches $q \approx 1.3 l$ (i.e., $R_{s} \approx 1.6 l$ ).

\section{b) Conditions for Merging and Ring Formation}

First, we examine the conditions for merging of the shock waves. As seen in $\S$ III, the collision of adiabatic shock waves leads to complete merging without producing a ring. Thus, we may safely give the condition for complete merging as

$$
t_{\text {coll }}<t_{\text {cool }}\left(z_{i}\right),
$$

where $t_{\text {coll }}$ is the time of the collision, and $t_{\text {cool }}\left(z_{i}\right)$ is the cooling time evaluated at $z=z_{i}$. Though $t_{\text {cool }}\left(z_{\text {coll }}\right)$ may be more adequate for this condition, we take $t_{\text {cool }}\left(z_{i}\right)$ for a simple numerical estimate. The expansion law of the adiabatic shock wave in an $\Omega=\Omega_{g}=1$ universe can be approximated as

$$
R_{s}(t)=1.1 \times 10^{11} E_{61}^{1 / 5} t^{2 / 5}\left(t_{u, i}+t\right)^{2 / 5} \mathrm{~cm},
$$

where $t_{u, i}$ is the cosmic time at $z=z_{i}$. The collision time $t_{\text {coll }}$ is given by $d=2 R_{s}\left(t_{\text {coll }}\right)\left(1+z_{\text {coll }}\right)$. Since the cooling time due to the inverse Compton process, which dominates at $z \gtrsim 10$, is given by $t_{\text {cool }}=7.67 \times 10^{19}\left(1+z_{i}\right)^{-4} \mathrm{~s}$, the condition (27) can be translated into the condition for $d$ at a given $z_{i}, d<$ 


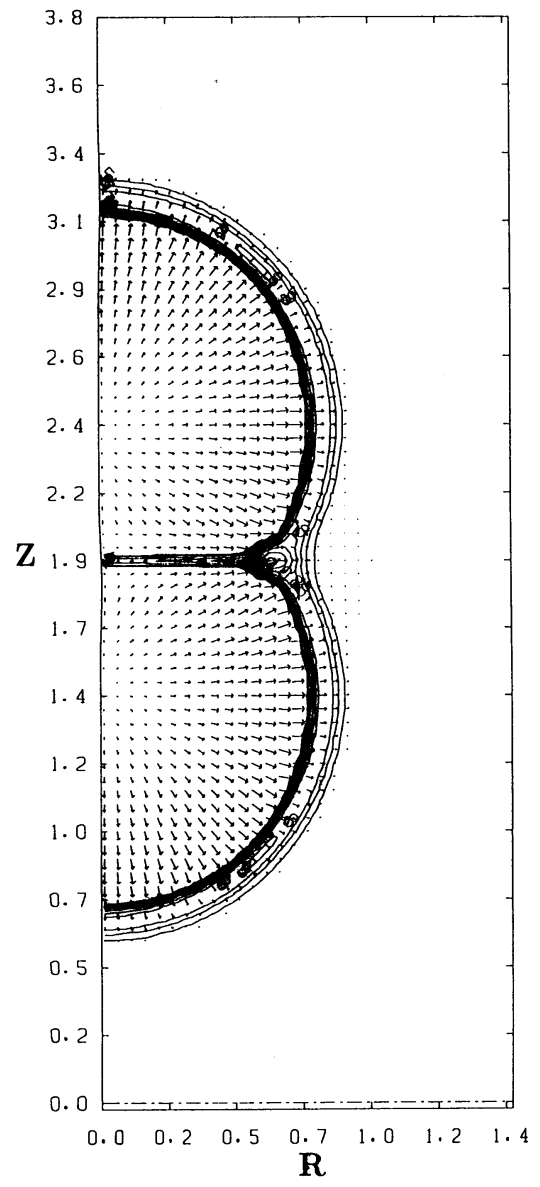

Fig. $9 a$

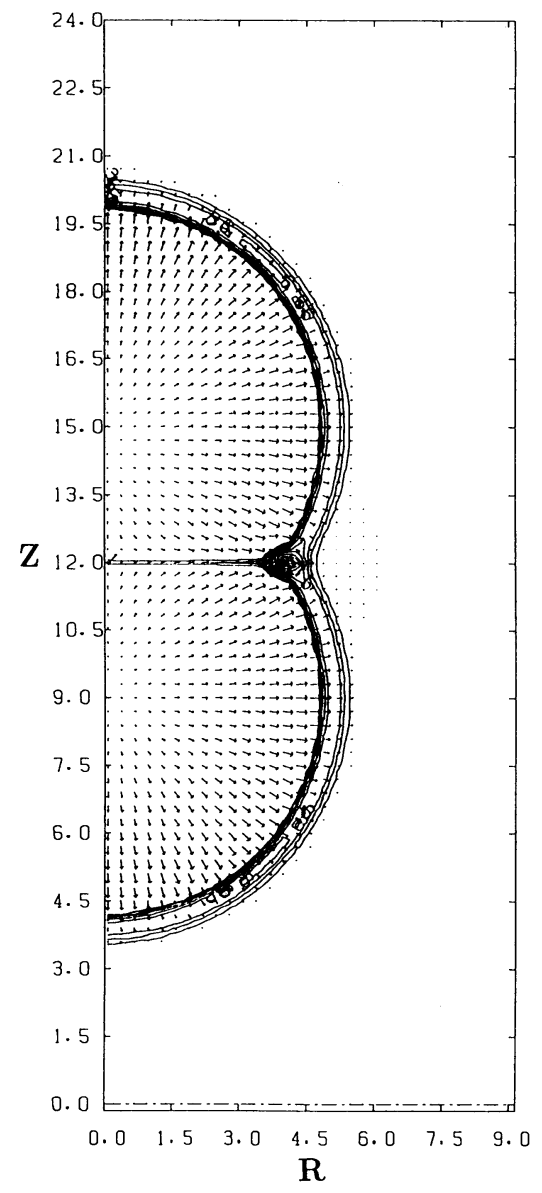

FIG. $9 b$

Fig. 9.-Dependence on the explosion energy. (a) The case of a smaller explosion energy: model E. Parameters are the same as in Fig. 4 except that the explosion energy $E_{i}=10^{59} \mathrm{ergs}$ and $d=2.4 \times(0.01)^{0.2} \mathrm{Mpc}$. The logarithm of the maximum level of the density contour is 1.018 . The maximum velocity is $3.9 \times 10^{1} \mathrm{~km} \mathrm{~s} \mathrm{~s}^{-1}$. (b) The case of a larger explosion energy: model F. Parameters are the same as in Fig. 4 except that the explosion energy $E_{i}=10^{63} \mathrm{ergs}$ and $d=2.4 \times(100)^{0.2} \mathrm{Mpc}$. The logerithm of the maximum level of the density contour is 1.424 . The maximum velocity is $2.8 \times 10^{2} \mathrm{~km} \mathrm{~s}^{-1}$.
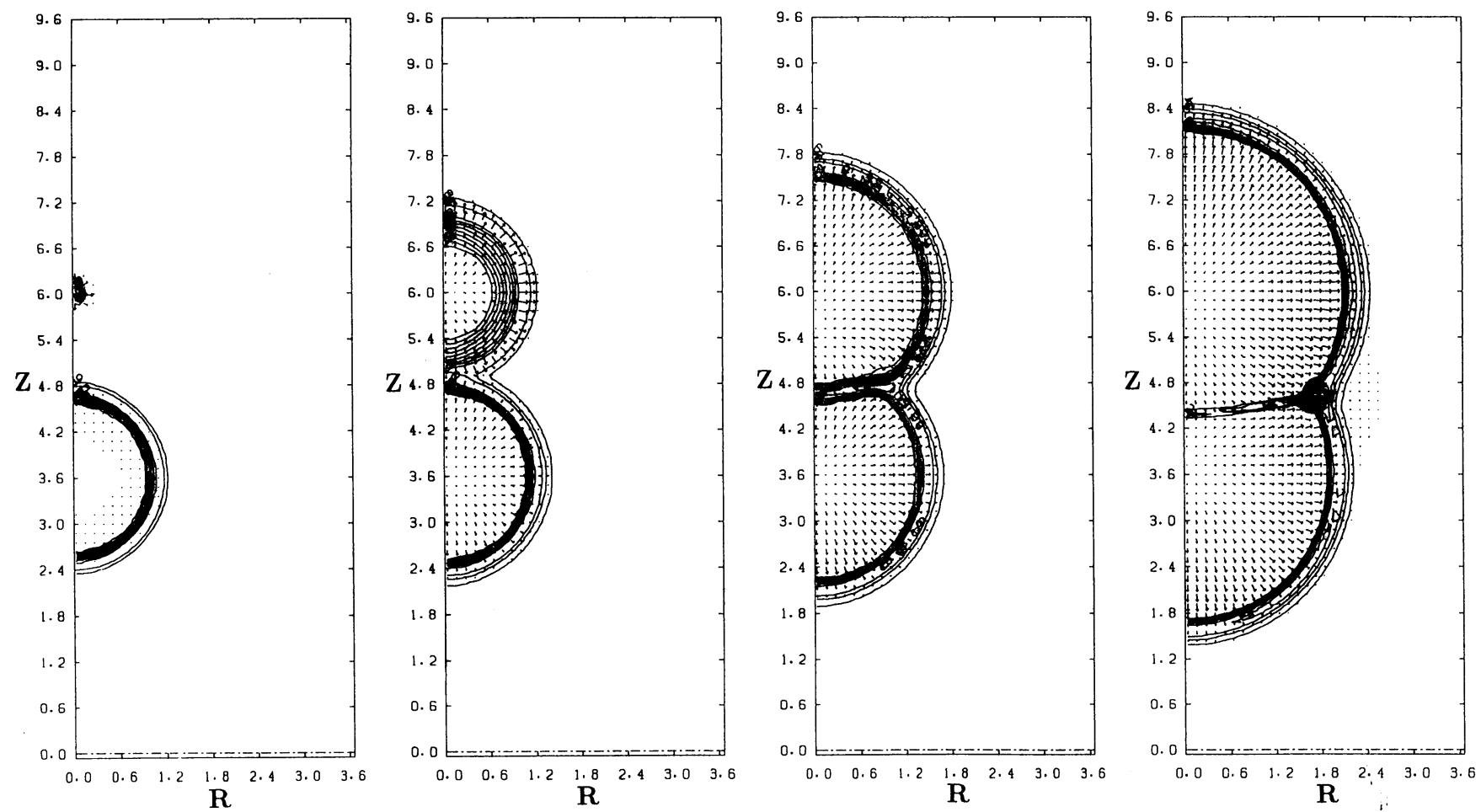

Fig. 10. Case of different explosion redshifts: model $\mathrm{G}\left(\Omega=1, \Omega=1, z_{i, 1}=20, z_{i, 2}=10, E_{i, 1}=E_{i, 2}=10^{61}\right.$ ergs, and $\left.d=2.4 \mathrm{Mpc}\right)$. The mesh size is $\Delta=0.06$ Mpc. The redshifts are 10.0, 7.6, 3.0, and 0.0 from left to right. The logarithms of the maximum levels of the density contours are $0.343,0.300,0.753$, and 1.154 , respectively. The maximum velocities are $7.1 \times 10^{3} \mathrm{~km} \mathrm{~s}^{-1}, 3.6 \times 10^{2} \mathrm{~km} \mathrm{~s}^{-1}, 1.7 \times 10^{2} \mathrm{~km} \mathrm{~s}^{-1}$, and $1.1 \times 10^{2} \mathrm{~km} \mathrm{~s}^{-1}$, respectively. 

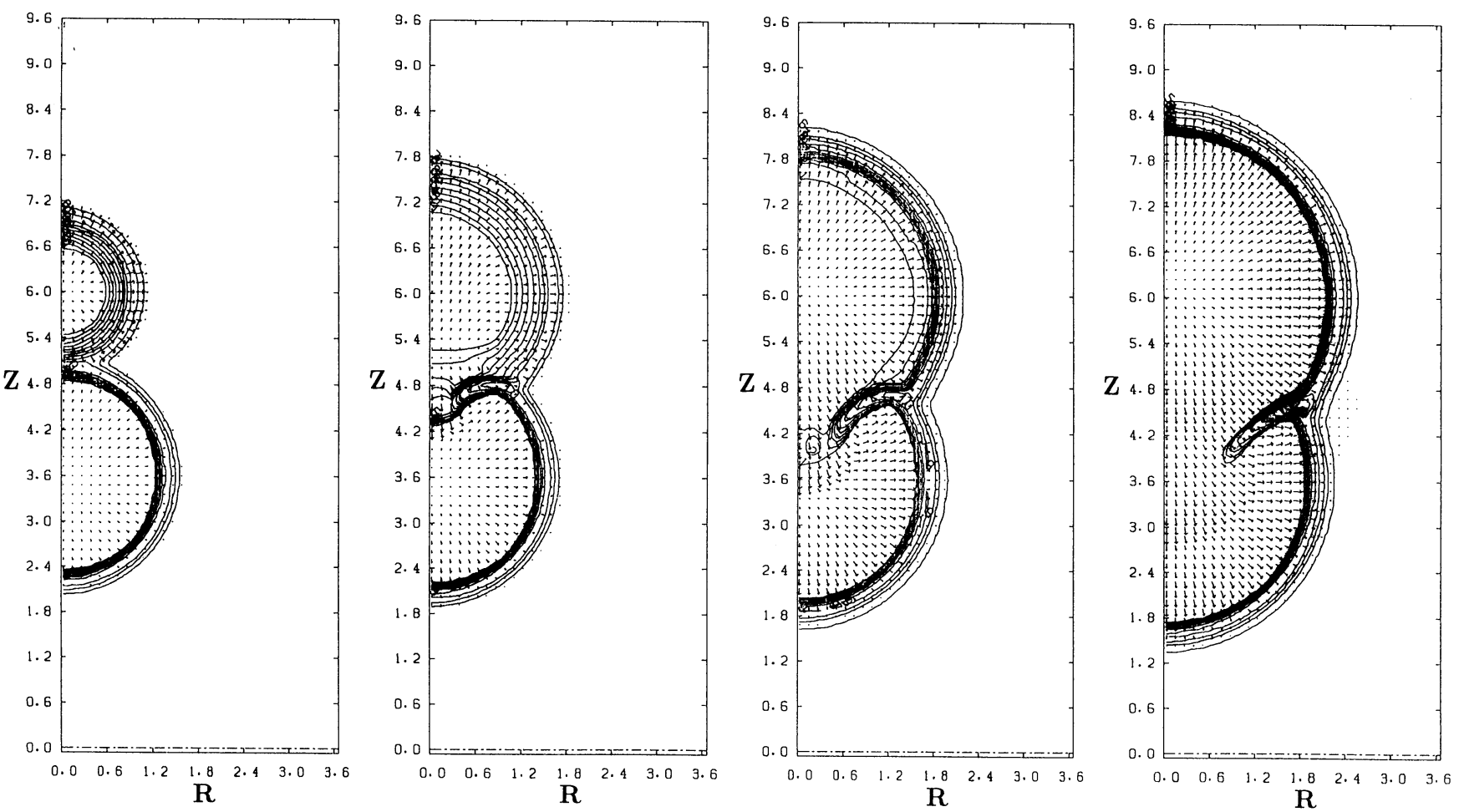

FIG. 11.-Case of different explosion redshifts: model $\mathrm{H}\left(\Omega=1, \Omega_{g}=1, z_{i, 1}=20, z_{i, 2}=5, E_{i, 1}=E_{i, 2}=10^{61}\right.$ ergs, and $\left.d=2.4 \mathrm{Mpc}\right)$. The mesh size is $\Delta=0.06$ Mpc. The redshifts are 4.2, 2.4, 1.0, and 0.0 from left to right. The logarithms of the maximum levels of the density contours are $0.452,0.674,0.737$, and 0.932 , respectively. The maximum velocities are $4.9 \times 10^{2} \mathrm{~km} \mathrm{~s}^{-1}, 2.6 \times 10^{2} \mathrm{~km} \mathrm{~s}^{-1}, 1.5 \times 10^{2} \mathrm{~km} \mathrm{~s}^{-1}$, and $1.0 \times 10^{2} \mathrm{~km} \mathrm{~s}^{-1}$, respectively.

$2 R_{s}\left(t_{\text {cool }}\right)\left[1+z\left(t_{\text {cool }}\right)\right]$. In fact, the condition $d<R_{s}\left(t_{\text {cool }}\right)$ $\left[1+z\left(t_{\text {coor }}\right)\right]$ seems to agree better with the numerical results except for higher $z_{i}$. The cooling of shells just after the collision leads to ring formation.

Next, we examine the conditions for ring formation. As seen in the previous subsection, a ring forms at the intersection point of outer shock waves by overtaking of colliding matter so

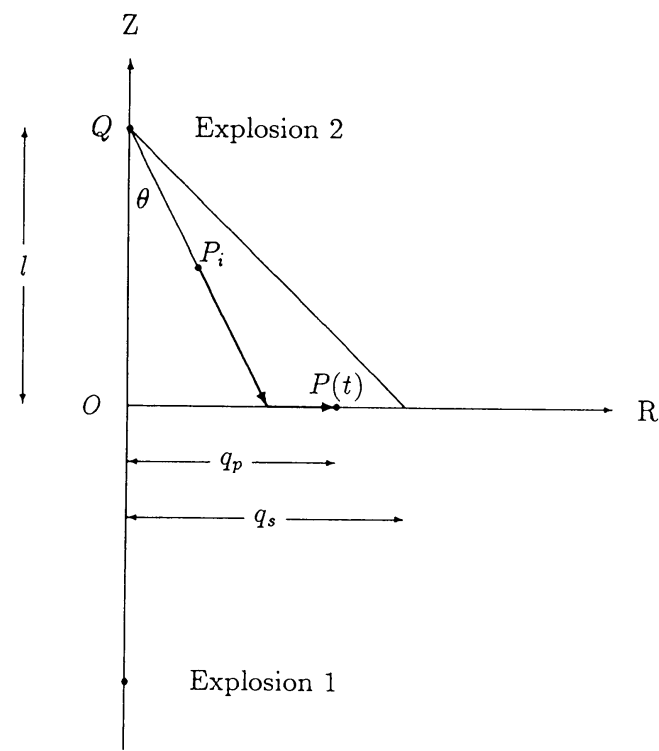

FIG. 12.- Schematic diagram of particle motions for inducing the ring formation. long as the matter has been cooled in the dense shells. Since the convergence occurs when the intersction point proceeds $\sim d / 2$ along the plane of the interaction, the condition for ring formation by $z=0$ will be given by

$$
d<a_{1} R_{s}(z=0) .
$$

The condition for the merging of cooled, dense shells by $z=0$ will be given by

$$
d<a_{2} R_{s}(z=0) .
$$

$R_{s}(z=0)$ is the shock radius at $z=0$ considering cooling and it can be obtained not analytically but numerically. The coefficients $a_{1}$ and $a_{2}$ are fitting parameters of $\sim O(1)$. Taking $a_{1}=$ 1.4 and $a_{2}=1.1$, the conditions (29) and (30) reproduce the numerical results as seen by the dash-dotted and dashed lines in Figure 14, respectively. The conditions obtained here are given for $E_{i}=10^{61}$ ergs, but the scaling law $d \rightarrow d\left(E_{i} / 10^{61}\right.$ ergs $)^{1 / 5}$ can be applied to different explosion energies. The reason why we can use the scaling law of $R_{s} \propto E_{i}^{1 / 5}$ is as follows: The thermal energy of the shock wave becomes negligible compared to its total energy at about the cosmic time scale $t_{u}$ (Bertschinger 1983), and the total energy remains approximately constant after cooling. Thus the total energy after cooling $E_{\text {cool }}$ can be written as $E_{\text {cool }} \approx E_{i} \exp (-b)$, where $b \approx t_{u} / t_{\text {cool }}$. When the Compton cooling is dominant, the cooling time $t_{\text {cool }}$ does not depend upon $E_{i}$. Therefore, $E_{\text {cool }} / E_{i}$ depends only very weakly on $E_{i}$, since the evolution of the shock wave after cooling is similar to that of an adiabatic shock wave whose initial energy is set to be $E_{\text {cool }}$, particularly, concerning the expansion law, the scaling $R_{s} \propto E_{\text {cool }}^{1 / 5} \propto E_{i}^{1 / 5}$ is achieved for fixed $z_{i}$. 


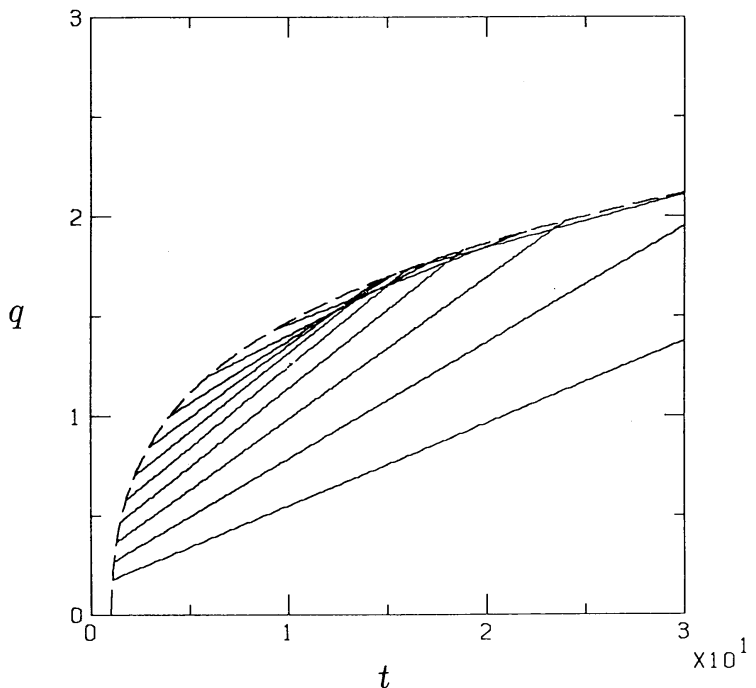

FIG. $13 a$

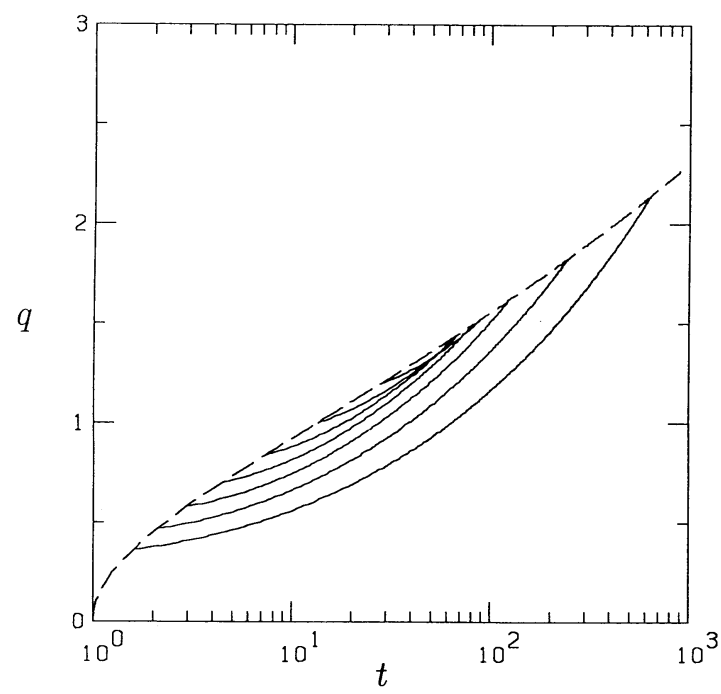

Fig. $13 b$

FIG. 13.-Trajectories of the colliding particles on the symmetry plane. (a) The case in the stationary medium. The expansion law of the shock wave is $R_{\mathrm{s}} \propto t^{0.25}$.

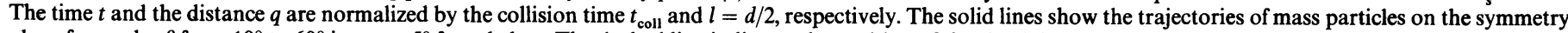

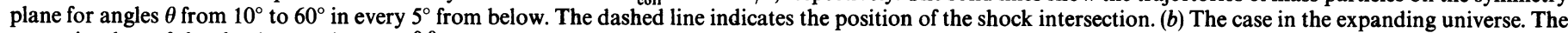

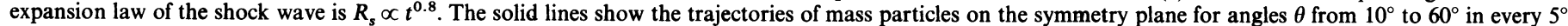
from below. The dashed line indicates the position of the shock intersection.

\section{c) Volume of Merged Bubbles}

In Table 1, we summarize the numerical results at $z=0$. When the two shock waves merge, the total volume of the merged shell $V_{\mathrm{sw}}$, their effective radius $R_{\mathrm{sw}} \equiv\left(3 V_{\mathrm{sw}} / 4 \pi\right)^{1 / 3}$, and

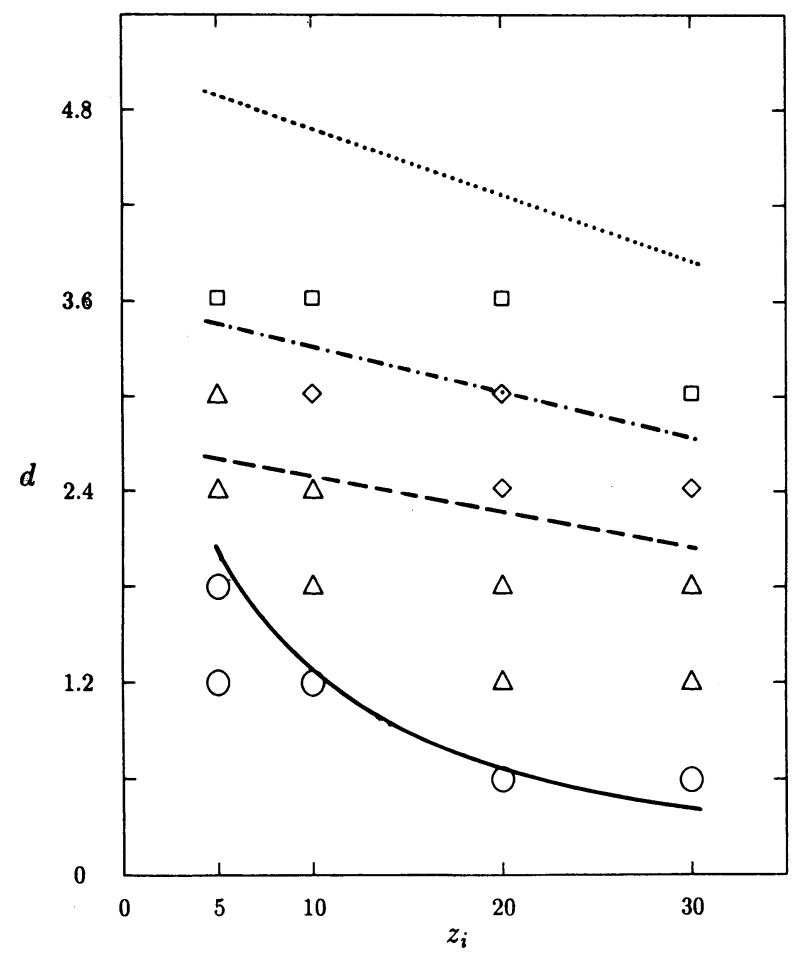

FIG. 14.-Analytically derived dependence of produced structure in the case of the $\operatorname{HD}$ model $\left(\Omega=1, \Omega_{g}=1\right.$, and $\left.E_{i}=10^{61} \mathrm{ergs}\right)$. Below the solid line, the shock waves merge, not forming a ring. Between the solid and dashed lines, the shock waves merge, forming a ring. Between the dashed and dash-dotted Hines, the shock waves do not merge with a ring. Between the dash-dotted and dotted lines, the shock waves do not merge without a ring. Above the dotted lime, the two shock waves do not collide each other by $z=0$. the ratio of the volume to that of a single explosion for the same $z_{i}$ and $E_{i}$ are tabulated. Corresponding values for single explosions are tabulated in Table 2 for comparison. In addition, it is indicated whether a ring is formed. When a ring structure is seen, the dimensionless maximum density enhancement in the ring $\kappa_{\max }=\rho_{\text {ring }} / \rho_{0}$ is shown.

The self-similar solution of the adiabatic cosmological shock wave predicts the radius of the shock wave with twice energy to be $2^{1 / 5}$ times as large as a single one. Thus, the volume of the bubble produced by merging of two shells is expected to be $2^{3 / 5}=1.52$ times as large. In the case of smaller $d$ 's, the volume ratios obtained in numerical calculations are $\sim 1.5$, which conforms to this expectation. However, with increasing $d$ the

TABLE 2

Numerical Results of a Single Explosion IN AN EXPANDING MEDIUM

\begin{tabular}{cccc}
\hline \hline$z_{i}$ & $N_{\text {shell }}$ & $V_{\text {sw }}$ & $R_{\text {sw }}$ \\
\hline \multicolumn{4}{c}{$\begin{array}{c}\text { A. HD Model } \\
\left(\Omega=\Omega_{g}=1, E_{i}=10^{61}\right.\end{array}$} \\
ergs, $\Delta=0.06 \mathrm{Mpc})$ \\
\hline $5 \ldots \ldots$ & $3.1 \times 10^{19}$ & 61.6 & 2.45 \\
$10 \ldots \ldots$ & $3.1 \times 10^{19}$ & 55.2 & 2.36 \\
$20 \ldots \ldots$ & $2.8 \times 10^{19}$ & 39.9 & 2.12 \\
$30 \ldots \ldots$ & $2.4 \times 10^{19}$ & 31.0 & 1.95 \\
\hline
\end{tabular}

B. LD Model

$\left(\Omega=\Omega_{g}=0.1, E_{i}=10^{61} \mathrm{ergs}, \Delta=0.1 \mathrm{Mpc}\right)$

\begin{tabular}{cccc}
$5 \ldots \ldots$ & $5.2 \times 10^{18}$ & 362.5 & 4.52 \\
$10 \ldots \ldots$ & $5.0 \times 10^{18}$ & 281.4 & 4.07 \\
$20 \ldots \ldots$ & $4.0 \times 10^{18}$ & 166.8 & 3.42 \\
$30 \ldots \ldots$ & $3.8 \times 10^{18}$ & 125.2 & 3.10 \\
\hline
\end{tabular}

C. DM Model

\begin{tabular}{cccc}
\multicolumn{4}{c}{$\left(\Omega=1, \Omega_{g}=0.1, E_{i}=10^{61}\right.$ ergs, $\left.\Delta=0.1 \mathrm{Mpc}\right)$} \\
\hline $5 \ldots \ldots$ & $4.1 \times 10^{18}$ & 227.0 & 3.78 \\
$10 \ldots \ldots$ & $3.8 \times 10^{18}$ & 164.2 & 3.40 \\
$20 \ldots \ldots$ & $3.5 \times 10^{18}$ & 77.5 & 2.64 \\
$30 \ldots \ldots$ & $2.8 \times 10^{18}$ & 48.3 & 2.26 \\
\hline
\end{tabular}


volume ratios considerably increase, because the merged volume is not determined by energy, but by simple overlapping of the two shells.

\section{d) Mass of the Rings}

As shown above, a ring is formed for appropriate $z_{i}$ and $d$. The absolute values of $\kappa_{\max }$ in Table 1 may not have physical meaning owing to the limited resolution of the calculations, but relative comparison of them will give the tendency of density enhancements in the rings.

It is rather difficult to estimate exactly the mass of a ring because of the ambiguity of identification of the ring region. In the HD models, the approximate values of ring masses for $E_{i}=10^{61}$ ergs are $M_{\text {ring }} \sim(2-3) \times 10^{12} M_{\odot}$; in the LD models, $M_{\text {ring }} \sim 10^{12} M_{\odot}$. These values are estimated in the following way. Suppose two cooled, dense shells having the same explosion redshift and energy collide. All colliding matter is assumed to concentrate to the point where the two shocks intersect to form a ring. Then, the mass of the ring is given by

$$
M_{R}=M_{T} \times \frac{\int_{\cos \eta}^{1} d(\cos \theta)}{\int_{-1}^{1} d(\cos \theta)}=\frac{M_{T}(1-\cos \eta)}{2},
$$

where $M_{T}$ is the total mass swept up into a single shell given by $M_{T}=(4 \pi / 3) R_{s}^{3} \rho_{\text {crit }} \Omega_{g}$, and $\eta$ is the angle between the symmetric axis and the direction to the point of the intersection given by $\cos \eta=d / 2 R_{s}$. Hence,

$$
\begin{aligned}
& M_{R}=\frac{2 \pi}{3} R_{s}^{3} \Omega_{g} \rho_{\text {crit }}\left(1-\frac{d}{2 R_{s}}\right)=5.8 \times 10^{11} \Omega_{g} h_{100}^{2}(1+z)^{3} \\
& \times\left(\frac{R_{s}}{1 \mathrm{Mpc}}\right)^{3}\left(1-\frac{d}{2 R_{s}}\right) M_{\odot} .
\end{aligned}
$$

In the HD and LD cases, the radius of the shell at $z=0$ is $R_{s} \approx 2.1 \mathrm{Mpc}$ and $3.4 \mathrm{Mpc}$, respectively, for $z_{i}=20$ and $E_{i}=$ $10^{61}$ ergs for instance. These give $M_{R} \approx 2.7 \times 10^{12} M_{\odot}$ and $1.1 \times 10^{12} M_{\odot}$ for $R_{s}=d$. Note that in the LD model the ring mass is comparatively large even for the low gas density.

\section{GRAVITATIONAL INSTABILITY OF THE RING}

The ring produced by the collision is expanding with the velocity of the outer shock waves. The problem of the gravitational instability of expanding rings may be replaced by that of cylinders stretching along their axis so long as the ring radius is much smaller than the curvature radius and the wavelength is small. The gravitational instability of an infinitely long, equilibrium cylinder has been investigated by linear stability analysis by many authors (Chandrasekhar and Fermi 1953; Stodolkiewicz 1963; Nagasawa 1987; see also Larson 1985), but that of stretching cylinders has never been examined. Here, we present a simple stability analysis using the energy principle similar to that by Ostriker and Cowie (1981) for an expanding spherical shell.

First we study the stability of an infinite cylinder at rest. Suppose a column with length $2 x$ along the axis is cut out from the infinite cylinder of radius $h$. If the sum of its thermal energy and gravitational energy is negative, we can expect this column may fragment out from the cylinder. The gravitational energy of the column is calculated by approximating it as a spheroid. When $x<h$, it is an oblate spheroid, whose gravitational energy per unit mass is given by

$$
W=-\frac{3}{5} \frac{m G}{h e} \sin ^{-1} e=-\frac{6 \pi}{5} \frac{x h G \rho}{e} \sin ^{-1} e,
$$

where $e$ is the eccentricity defined by $x^{2}=h^{2}\left(1-e^{2}\right)$ and $m=\pi h^{2} \cdot 2 x \rho$ is the mass of the column. When $x>h$, it is prolate and its gravitational energy is given by

$$
W=-\frac{3}{10} \frac{m G}{x e} \log \left(\frac{1+e}{1-e}\right)=-\frac{3 \pi}{5} \frac{h^{2} G \rho}{e} \log \left(\frac{1+e}{1-e}\right),
$$

where $h^{2}=x^{2}\left(1-e^{2}\right)$.

From the equation of motion for an equilibrium cylinder, the thermal energy per unit mass of the column is estimated as $T=b_{1} G \rho h^{2}$, where $b_{1}$ is a numerical constant of order unity. If $W+T<0$, the instability will occur. This condition obtained here is similar to that obtained from a linear stability analysis (e.g., Larson 1985) except for numerical factors. In addition $(W+T) / x^{2}$ has a minimum value $-b_{2} G \rho$, where $b_{2}$ is a constant of order unity.

Next, we obtain the instability criterion for an expanding ring using the above results. When the ring expands with the velocity $V_{R}$, the velocity structure along the axis of the column cut out from the ring is given by $V_{H}=V_{R}(y / R)$, where $y$ is the distance from the cente: of the column and $R$ is the curvature radius of the ring from the symmetric axis. Thus, the kinetic energy per unit mass of the column is

$$
K=\frac{1}{2} \int_{-x}^{x} \frac{\rho V_{H}^{2} \pi h^{2} d y}{m}=\frac{1}{6}\left(\frac{V_{R}}{R}\right)^{2} x^{2} .
$$

Thus, the criterion that the ring has unstable modes is expressed as

$$
\left(\frac{W+T}{x^{2}}\right)_{\min }+\frac{K}{x^{2}}=-b_{2} G \rho+\frac{1}{6}\left(\frac{V_{R}}{R}\right)^{2}<0 .
$$

Supposing that $R \propto t^{\alpha}$, this condition leads to

$$
\frac{\alpha}{t}<\left(6 b_{2} G \rho\right)^{1 / 2} \text {. }
$$

In a flat universe $(\Omega=1), \alpha \sim 0.8$ for $R \sim R_{s}$ and taking account of the density of the ring $\rho=\kappa \Omega_{g} \rho_{\text {crit }}=1.879$ $\times 10^{-29} \Omega_{g} h_{100}^{2} \kappa(1+z)^{3}$, where $\kappa$ is the density enhancement in the ring, we find from equation (37)

$$
\kappa>\frac{0.64 \pi}{b_{2} \Omega_{g}} .
$$

This criterion is easily satisfied in the HD models, $\kappa \gtrsim 20$ in the present results, and the ring will fragment. In a low-density universe $(\Omega \ll 1), \alpha=1$, and the criterion is rewritten as

$$
\kappa>\frac{4 \pi}{9 b_{2}} \frac{1}{\Omega_{g}(1+z)} .
$$

This condition does not seem to be satisfied in the LD models. However, because of the limited resolution of our calculations and the crudeness of the instability criterion, it is quite possible that the ring may fragment.

\section{LARGE-SCALE STRUCTURES}

Here, we discuss the formation of large-scale structures by cosmic explosions using the numerical results. First, we express the conditions for merging of shells and ring formation in terms of the explosion energy and the number density of exploding seeds. The mean distance between the seeds $\bar{r}$ is given using their present number density $n_{s}$ as follows:

$$
\bar{r}=\left(\frac{4 \pi}{3} n_{s}\right)^{-1 / 3}=0.62\left(\frac{n_{s}}{1 \mathrm{Mpc}^{-3}}\right)^{-1 / 3} \mathrm{Mpc} \text {. }
$$


If the shock waves expand independently and the merging of them is not significant, $\bar{r}$ can be interpreted as the mean radius of the bubbles. Using the mean separation $d=2 \bar{r}$, we express the conditions of collision, merging, and ring formation at a fixed $z_{i}$ in the $E_{i}-n_{s}$ plane, where we make use of the conditions obtained by simulations and the scaling laws for different $E_{i}$ as in Figure 15.

Further, we plot there other related important quantities: the mean efficiency of energy release, the Compton $y$ parameter, and the degree of the anisotropy of the cosmic microwave background radiation (CBR) produced by explosions.

\section{a) Mean Efficiency of Energy Release}

If we assume the energy is released from baryonic matter, the mean efficiency of energy release $\epsilon$ is defined by

$$
\epsilon=\frac{E_{i} n_{s}}{\rho_{g} c^{2}}=2.01 \times 10^{-5}\left(\frac{E_{i}}{10^{61} \mathrm{ergs}}\right)\left(\frac{n_{s}}{1 \mathrm{Mpc}^{-3}}\right) \Omega_{g}^{-1} h_{100}^{-2} .
$$

If the energy is produced by nuclear reactions, $\epsilon$ is at most $\sim 10^{-2}$, and usually $\epsilon \sim 10^{-4}$ in the case of supernova explosions of massive stars. This efficiency is calculated with respect to all the baryonic matter, and hence it must be much higher if only a part of the baryonic matter participates in the energy retease.

\section{b) Compton y-Parameter}

The Compton $y$-parameter measures the degree of the distortion of the CBR spectrum from the Planck one by inverse Compton scattering. In addition, the distorted fraction of the radiation energy density is given using the $y$-parameter as follows:

$$
\frac{\Delta u_{r}}{u_{r}}=e^{4 y}-1 \simeq 4 y,
$$

where the radiation energy density is $u_{r}=a T_{r}^{4}=4.02$ $\times 10^{-13}\left(T_{r} / 2.7 \mathrm{~K}\right)^{4}(1+z)^{4}$. If we assume all the released energy is instantaneously converted to the radiation energy, i.e., $\Delta u_{r}=\epsilon \rho_{g}(1+z)^{3} c^{2}$, which will be approximately justified in the Compton cooling era $(z \gtrsim 10)$, the $y$-parameter is given by

$$
y=1.05 \times 10^{4} \Omega_{g} h_{100}^{2} \epsilon(1+z)^{-1} .
$$

The Nagoya-Berkeley rocket observation discovered a distortion of the CBR at submillimeter wavelengths (Matsumoto et al. 1988). If this distortion is due to the inverse Compton scattering, the $y$-parameter is estimated as $y \sim 0.02$ (Hayakawa $e t$ al. 1987; Yoshioka and Ikeuchi 1987). Then, the mean efficiency of the energy release must be

$$
\epsilon=2 \times 10^{-6}(y / 0.02) \Omega_{g}^{-1} h_{100}^{-2}\left(1+z_{i}\right) .
$$

\section{c) Anisotropy of the Cosmic Background Radiation}

Here, we roughly estimate the anisotropy of CBR produced by explosions. When the explosion sources are distributed randomly in space, the expected Sunyaev-Zeldovich anisotropy is given by (Vishniac and Ostriker 1986; Yoshioka and Ikeuchi 1987, 1988)

$$
\left\langle\left(\frac{\Delta T}{T}\right)^{2}\right\rangle=\int\left[\frac{2}{3}\left(\frac{\sigma_{\mathrm{T}}}{A_{b}}\right)\left(\frac{E_{\mathrm{th}}}{m_{e} c^{2}}\right)\right]^{2} n_{s}(1+z)^{3} A_{b} c d t,
$$

where $\sigma_{\mathrm{T}}$ is the Thomson cross section, $m_{e}$ is the electron mass, $E_{\text {th }}$ is the thermal energy per explosion, and $A_{b}$ is the cross section of the observing beam. When the Compton cooling is efficient, i.e., $z \gtrsim 10$, the main contribution to the integral comes from the time interval $\Delta t \simeq t_{\text {cool }}=7.67 \times 10^{19}(1+z)^{-4}$ s. When $z \gg 1, A_{b} \simeq \pi\left[\left(c / H_{0}\right) \Delta \theta \Omega^{-1}(1+z)^{-1}\right]^{2}$, where $\Delta \theta$ is the beam angle. Hence, the expected anisotropy $\Delta$ is given by

$$
\begin{aligned}
\Delta=\left\langle\left(\frac{\Delta T}{T}\right)^{2}\right\rangle^{1 / 2} & =3.16 \times 10^{-4}\left(\frac{E_{i}}{10^{61} \mathrm{ergs}}\right) \\
& \times\left(\frac{n_{s}}{1 \mathrm{Mpc}^{-3}}\right)^{1 / 2} h_{100}\left(\frac{\Delta \theta}{1^{\prime}}\right)^{-1}(1+z)^{1 / 2},
\end{aligned}
$$

where we assume $E_{\mathrm{th}}=E_{i}$.

The most stringent upper limits to anisotropy are $1.9 \times 10^{-5}$ on $\Delta \theta=0.75$ (Uson and Wilkinson 1984) and $2.1 \times 10^{-5}$ on $\Delta \theta=1^{\prime} .8$ (Readhead et al. 1989). However, we conservatively take the upper limit to be $\sim 10^{-4}$ in consideration of the roughness of the present estimate and possible correlation between the beams.

\section{d) Summary}

Figure $15 a$ shows the conditions of merging or ring formation, as well as the mean efficiency $\epsilon$, the Compton $y$ parameter, and the expected CBR anisotropy $\Delta$ in the HD model with $z_{i}=20$.

The model satisfying the bubble filling factor $f \sim 1$, defined by $f=R_{s}^{3} n_{s}(1+z)^{3}$, and $\Delta \sim 10^{-4}$ corresponds to $E_{i} \sim$ $10^{60-61}$ ergs and $\bar{r} \sim 1-2 \mathrm{Mpc}$. This scale length seems much smaller than that of the observed bubbles. If the CBR anisotropy can be erased by subsequent Thomson scattering, explosions with $E_{i} \sim 10^{66}$ ergs can make bubbles with $\bar{r} \sim 20 \mathrm{Mpc}$ and produce the CBR distortion with $y \sim 0.01$, although the explosion redshift must be greater than $\sim 100$.

Figure $15 b$ shows the relations in the LD model, in which large bubbles are easy to make. The model satisfying $f \sim 1$ and $\Delta \sim 10^{-4}$ corresponds to $E_{i} \sim 10^{62-63}$ ergs and $\bar{r} \sim 7-8 \mathrm{Mpc}$, which is larger than in the MD model but still not satisfactory. If the CBR anisotropy can be erased out by Thomson scattering, explosions with $E_{i} \sim 10^{66}$ ergs make bubbles with $\bar{r} \sim 20 \mathrm{Mpc}$. The CBR distortion with $y \sim 0.01$ is also produced. The required efficiency of energy release is $\epsilon \sim 10^{-4}$.

In sum, explosions with $E_{i} \sim 10^{66}$ ergs can make the observed large bubbles, producing the CBR distortion discovered by Matsumoto et al. (1988) at the same time, although subsequent Thomson scattering and large efficiency of energy release are needed (Yoshioka and Ikeuchi 1987).

A completely different situation may be possible. Merging of multiple shock waves may make the observed large bubbles. For instance, explosions with $E_{i} \sim 10^{60-61}$ ergs and $n_{s} \sim 1-20$ $\mathrm{Mpc}^{-3}$ will result in significant merging of shock waves without violating the CBR isotropy. This energy scale is moderate in galaxy evolution. Since the radius of a single shock wave is $\sim 1 \mathrm{Mpc}$, we must examine the merging process of such small shock waves.

\section{DISCUSSION}

We summarize the results of the calculations:

1. We investigated the collision process of two cosmological shock waves produced by explosions by two-dimensional numerical hydrodynamical calculation.

The structures resulting from the collisions can be classified into four categories by their global appearances; complete 


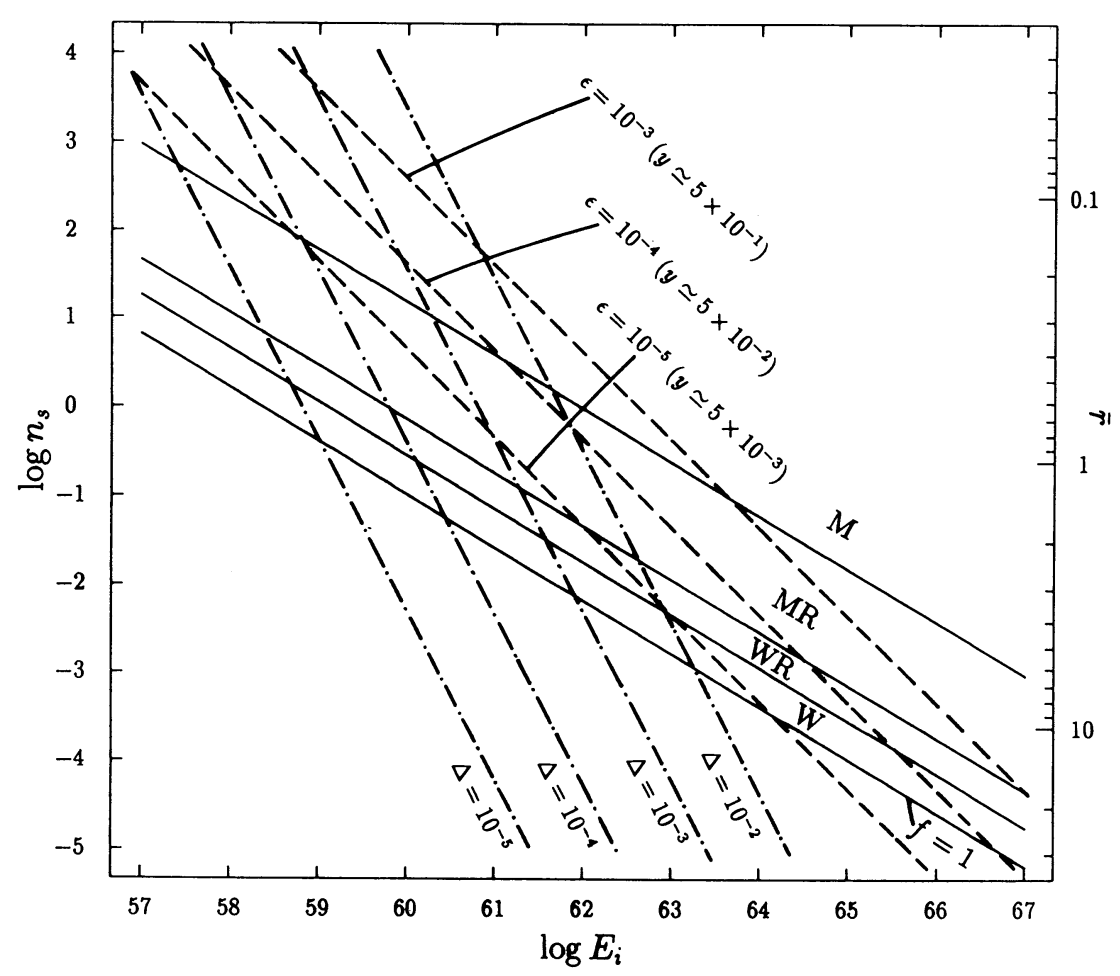

FIG. $15 a$

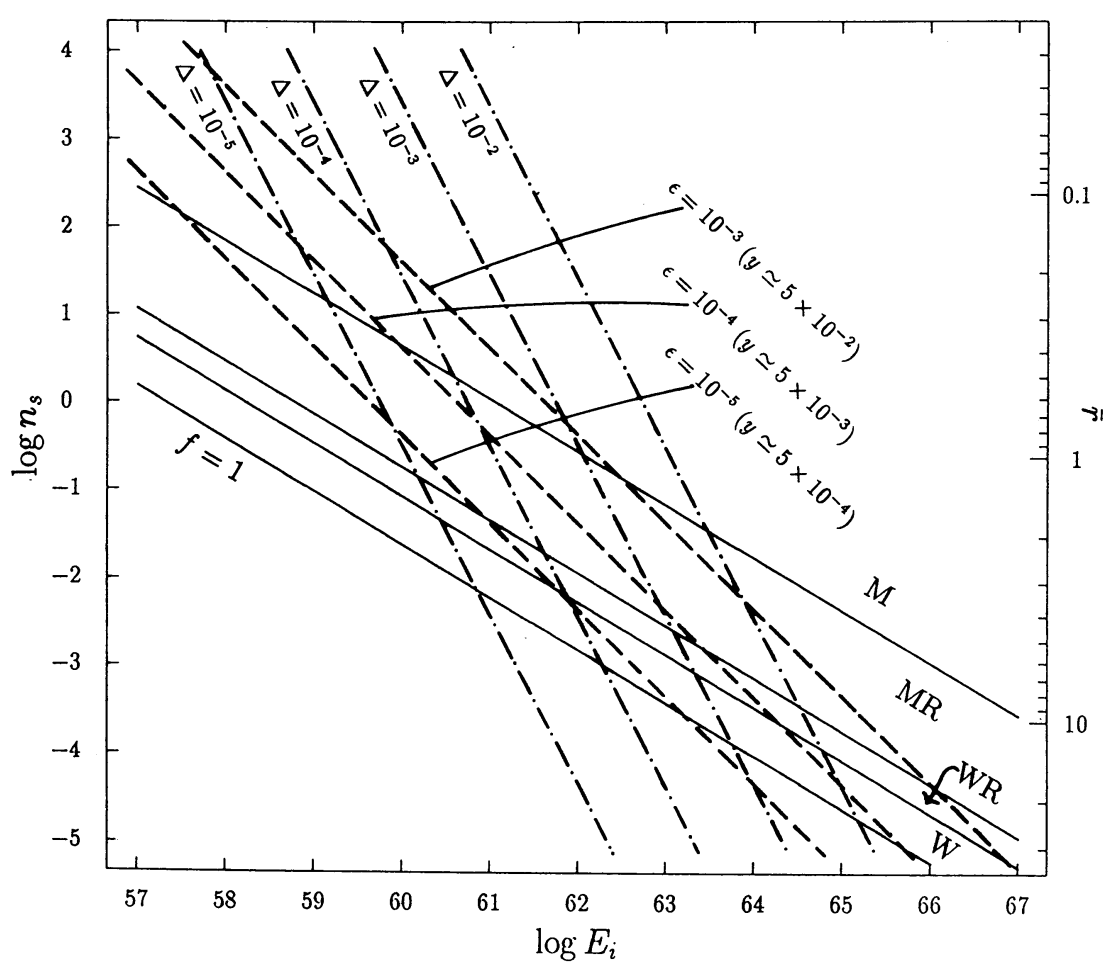

Fig. $15 b$

FIG. 15.-Constraints on the formation of large-scale structures in the explosion model. (a) The HD model $\left(\boldsymbol{\Omega}=\boldsymbol{\Omega}_{g}=1\right)$ and the explosion redshift $z_{i}=20$. The solid lines indicate the conditions of merging or ring formation. The dashed lines represent the efficiency of energy release $\epsilon$ as well as the Compton $y$-parameters. The dash-dotted lines represent the expected anisotropy of the cosmic background radiation in the scale $\Delta \theta=1^{\prime}$. (b) The same as in $(a)$ but for the LD model $(\Omega=1$ $\left.\Omega_{g}=0.1\right)$. 
merging case, merging case with a dense ring, nonmerging case with a ring, and nonmerging separated by a dense wall.

2. From calculations by varying various parameters, we obtained the parameter dependence of the structure formed. In particular, we classified the resultant structures in the plane of the explosion redshift $z_{i}$ and the comoving distance between the two explosions $d$. Moreover we discussed the dependence on the explosion energy $E_{i}$ by using a scaling law. We also calculated the evolution in the cases that the explosion redshifts are different.

3. We made clear the physical cause of the ring formation as a hydrodynamical process. In addition, we explored the conditions for merging and ring formation and obtained their simple semianalytical expressions for them.

4. From the analysis of the gravitational instability of an expanding ring, we concluded that in the HD model, the ring is gravitationally unstable, giving rise to the fragments.

5. In the framework of the explosion model, explosions with $E_{i} \sim 10^{62-63}$ ergs can make bubble-like structures with a typical void diameter $\sim 10 \mathrm{Mpc}$ consistent with the CBR anisotropy and distortion. If the CBR anisotropy has been smoothed out by subsequent Thomson scattering, formation of larger voids is possible. The required efficiency of energy release is $\epsilon \sim 10^{-4}$.

Różyczka et al. found similar phenomena to the results presented in this paper in a situation without self-gravity or cosmic expansion: they showed that $(a)$ in an adiabatic case, a local density maxima occurs behind the shock intersection, but it is not maintained for a long time and the two shock waves merge, and $(b)$ in a cooling case, a dense ring forms behind the shock intersection.

Finally, we discuss the formation of large-scale structures by comparing the present results with the simple treatment by Yoshioka and Ikeuchi (1989), in which we model the cosmological structures by utilizing the three-dimensional Voronoi tessellation. The tessellation is made by a simple Monte Carlo method where the mass distribution is represented by particles. We take three types of models for the structures produced by the collisions: (1) the mass particles are distributed on the surfaces of the Voronoi polyhedra, (2) the mass particles on the surfaces are concentrated to the edges of the Voronoi polyhedra, and (3) the mass particles are concentrated to the vertices of the Voronoi polyhedra. The resultant structures are compared with the real structure by calculating the correlation functions of mass particles and the Voronoi vertices. The correlation function of the Voronoi vertices, which we may regard as clusters of galaxies, is shown to follow a power law whose index $\sim-2$.

In the Voronoi tessellation, the complete merging of the shock waves is not considered, i.e., each seed belongs to a single cell. This will be valid for the case of high- $z_{i}$ explosions and/or small number density of explosion seeds, since the collisions of young shock waves are rare. The numerical results obtained here will have the following correspondences to the above models. For fixed $z_{i}$, when $d$ is sufficiently large, a wall is produced in the colliding plane, which corresponds to the above model (1). When $d$ has a moderate value, a dense ring is produced in the intersection of the shock waves, and the wall disappears. This corresponds to the above model (2). Although our two-dimensional calculation cannot give a definite answer to collisions of more than two shock waves, we may expect high-density spots will be formed at the intersection points among plane shocks and rings. This will correspond to the above model (3). In fact, the real structures produced by collisions of many shock waves will be a mixture of these three models.

From the above, we may imagine the evolutionary sequence of the universe. At $z \gtrsim 10$, explosions with energy $\sim 10^{63}$ ergs occur and produce shock waves, which sweep up primordial gas into spherical shells. Small- $d$ collisions of the shock waves form larger merged shells. In these shells, objects with relatively small mass such as Ly $\alpha$ clouds and dwarf galaxies formed by gravitational instabilities and/or hydrodynamical instabilities. Moderate- $d$ collisions first produce high-density walls, which gradually concentrate to rings. In walls or rings, objects with mass comparable to typical galaxies are produced. Collisions of rings and shells make very massive objects in their intersections, where clusters of galaxies form. The present universe is a mixture of these structures. The universe shows a bubble-like appearance, where rings and walls still dominate. This requires the present bubble filling factor $f \sim 1$, as also pointed out by other authors (Weinberg, Ostriker, and Dekel 1989; Ostriker and Strassler 1989).

The above scenario also reproduces the correlation of objects on various scales. The structures dominated by rings and walls correspond to the galaxies of the correlation function $\xi_{g}$ with the power index $\sim-1.5$. Objects with relatively small mass such as Ly $\alpha$ clouds and dwarf galaxies which formed in the shells only have weak correlations. Clusters of galaxies, which formed in intersection spots of rings and shells, are rare objects, having the correlation function with the power index -2 (Yoshioka and Ikeuchi 1989; Weinberg, Ostriker, and Dekel 1989; van de Weygaert and Icke 1989).

The authors would like to thank K. Tomisaka for a helpful discussion and their colleagues in the Division of Theoretical Astrophysics for valuable comments. Numerical calculations were performed by Facom-M780 at National Astronomical Observatory and Facom-VP200 at the Institute of Space and Astronautical Science.

This work was supported in part by Grant-in Aid for Encouragement of Young Scientists from the Ministry of Education, Science and Culture (01790177). One of the authors (S. Y.) also acknowledges the JSPS Fellowship for Japanese Scientists for financial aid.

\section{REFERENCES}

Alcock, C., Fuller, G. M., and Mathews, G. J. 1987, Ap. J., 320, 439.

Applegate, J. H., Hogan, C. J., and Scherrer, R. J. 1987, Phys. Rev., D35, 1151. Bahcall, N. A., and Soneira, R. A. 1983, Ap. J., 270, 20.

Bertschinger, E. 1983, $A$ p. J., 268, 17.

Black, D. C., and Bodenheimer, P. 1975, Ap. J., 199, 619

Bodenheimer, P., York, H. W., and Tenorio-Tagle, G. 1984, Astr. Ap., 138, 215. Chandrasekhar, S., and Fermi, E. 1953, Ap. J., 118, 116.

da Costa, L. N., et al. 1988, Ap. J., 327, 544.

de Lapparent, V., Geller, M. J., and Huchra, J. P. 1986, Ap. J. (Letters), 302, L1.

Giovanelli, R., and Haynes, M. P. 1985, A.J., 90, 2445.
Hawley, J. F., Smarr, L. L., and Wilson, J. R. 1984, Ap. J. Suppl., 55, 211.

Hayakawa, S., Matsumoto, T., Matsuo, H., Murakami, H., Sato, S., Lange, A. E., and Richards, P. L. 1987, Pub. Astr. Soc. Japan, 39, 941.

Hogan, C. 1984, Ap. J. (Letters), 284, L1.

Huchra, J. P., Geller, M. J., de Lapparent, V., and Burg, R. 1988, in Large Scale Structure of the Universe, ed. J. Audouze, M.-C. Pelletan, and A. Szalay (Dordrecht: Kluwer), p. 105.

Ikeuchi, S. 1978, Pub. Astr. Soc. Japan, 30, 563.

1981, Pub. Astr. Soc. Japan, 33, 211.

Ikeuchi, S., Tomisaka, K., and Ostriker, J. P. 1983, Ap. J., 265, 583. 
Jones, E. M., Smith, B. W., Straka, W. C., Kodis, J. W., and Guitar, H. 1979, Ap. J.;232, 129

Larson, R. B. 1985, M.N.R.A.S. $214,379$.

Mac Low, M.-M., and Shull, J. M. 1986, Ap. J., 302, 585.

InI Matsumoto, T., Hayakawa, S., Matsuo, H., Murakami, H., Sato, S., Lange,

$1 \because$ A. E., and Richards, P. L. 1988, Ap.J., 329, 567

Nagasawa, M. 1987, Prog. Theoret. Phys., 77, 635

Norman, M. L., and Winkler, K.-H. A. 1986, in Astrophysical Radiation Hydrodynamics, ed. K.-H. A. Winkler, and M. L. Norman (Dordrecht: Reidel), p. 187

Ostriker, J. P., and Cowie, L. L. 1981, Ap. J. (Letters), 243, L127.

Ostriker, J. P., and Strassler, M. J. 1989, Ap. J., 338, 579.

Ostriker, J. P., and Thompson, C. 1987, Ap. J., (Letters), 323, L97.

Ostriker, J. P., Thompson, C., and Witten, E. 1986, Phys. Letters B, 280, 231.

Pierre, M., Shaver, P. A., and Iovino, A. 1988, Astr. Ap., 197, L3.

Readhead, A. C. S., Lawrence, C. R., Myers, S. T., Sargent, W. L. W., Hard-

ebeck, H. E., and Moffet, A. T. 1989, Ap. J., 346, 566.

Różyczka, M. 1985, Astr. Ap., 143, 59.

Różyczka, M., Tenorio-Tagle, G., and Bodenheimer, P. 1986, Astr. Ap., 167, 120.

SATORU IKEUCHI: Division of Theoretical Astrophysics, National Astronomical Observatory, Mitaka, Tokyo 181, Japan

SATOSHI YoshioKa: Department of Physics, Tokyo University of Mercantile Marine, Koto, Tokyo 135, Japan
Saarinen, S., Dekel, A and Carr, B, 1987, Nature, 325, 598.

Shandarin, S. F. 1980, Astrofizika, 16, 769.

Shapiro, P. R., and Kang, H. 1987, Ap. J., 318, 32

Shapiro, P. R., and Struck-Marcell, C. 1985, Ap. J. Suppl., 57, 205.

Stodolkiewicz, J. S. 1963, Acta Astr., 13, 30.

Uson, J. M., and Wilkinson, D. T. 1984, Ap. J., 283, 471.

van de Weygaert, R., and Icke, V. 1989, Astr. Ap., 213, 1.

van Leer, B. 1977, J. Comp. Phys., 23, 276.

Vishniac, E. T., and Ostriker, J. P. 1986, Soc. Italiana Fis., 1, 157.

Vishniac, R. T., Ostriker, J. P., and Bertschinger, E. 1985, Ap. J., 291, 399.

Weinberg, D., Ostriker, J. P., and Dekel, A. 1989, Ap. J., 336, 9.

Witten, E. 1984, Phys. Rev., D30, 272.

Yang, J., Turner, M. S., Steigmann, G., Schramm, D. N., and Olive, K. A. 1984 $J ., 281,493$

Yoshioka, S., and Ikeuchi, S. 1987, Ap. J. (Letters), 323, L7.

1988, Pub. Astr. Soc. Japan, 40, 383

. 1989, Ap. J., 341, 16.

Zel'dovich, Ya. B., and Sunyaev, R. A. 1969, Ap. Space Sci., 4, 301 